\title{
Global classical solution for a 3D viscous liquid-gas two-fluid flow model in a half-space
}

Jing Yang ${ }^{1}$, Zilai Li ${ }^{2 *}$ and Li Fang ${ }^{3}$

\section{"Correspondence:} lizilai0917@163.com

25chool of Mathematics and Information Science, Henan Polytechnic University, Jiaozuo, 454000, P.R. China

Full list of author information is available at the end of the article

\begin{abstract}
In this paper, we consider the three-dimensional viscous liquid-gas two phase flow model in a half-space $\Omega=\left\{x \in \mathbb{R}^{3}: x_{3}>0\right\}$, where the initial vacuum is allowed. We prove the existence of the global classical solutions when the energy of the initial data is small enough.
\end{abstract}

MSC: 76T10; 76N10; 35L65

Keywords: viscous liquid-gas two-fluid flow model; classical solution; vacuum; a half-space

\section{Introduction}

We consider a 3D viscous liquid-gas two-fluid flow model in the following form:

$$
\left\{\begin{array}{l}
m_{t}+\operatorname{div}(m u)=0, \\
n_{t}+\operatorname{div}(n u)=0, \\
(m u)_{t}+\operatorname{div}(m u \otimes u)+\nabla P(m, n)=\mu \Delta u+(\lambda+\mu) \nabla \operatorname{div} u, \quad x \in \Omega, t>0,
\end{array}\right.
$$

the half-space $\Omega=\left\{x \in \mathbb{R}^{3}: x_{3}>0\right\}$ and the initial boundary conditions are

$$
\begin{aligned}
& \left.(m, n, u)\right|_{t=0}=\left(m_{0}, n_{0}, u_{0}\right), \quad x \in \Omega, \\
& \left(u^{1}(x), u^{2}(x), u^{3}(x)\right)=\beta\left(u_{x_{3}}^{1}, u_{x_{3}}^{2}, 0\right), \quad \beta>0, \text { on } \partial \Omega, \\
& (m, n, u)(x, t) \rightarrow(\tilde{m}, \tilde{n}, 0), \quad \text { as }|x| \rightarrow \infty,(x, t) \in \Omega \times(0, T) .
\end{aligned}
$$

Here $\tilde{m}, \tilde{n}$ are positive constants. The variables $m=\alpha_{l} \rho_{l}, n=\alpha_{g} \rho_{g}, u=\left(u^{1}, u^{2}, u^{3}\right)$, and $P=$ $P(m, n)$ denote the liquid mass, the gas mass, the velocity of the liquid and the gas, and the common pressure for both fluids, respectively; $\mu$ and $\lambda$ denote the two Lamé coefficients, which are assumed to satisfy $\mu>0,2 \mu+3 \lambda \geq 0$. The other unknown variables $\rho_{l}$ and $\rho_{g}$ denote the liquid and gas densities, satisfying the equations of state $\rho_{l}=\rho_{l, 0}+\frac{P-P_{l, 0}}{a_{l}^{2}}$ and $\rho_{g}=\frac{P}{a_{g}^{2}}$, where $a_{l}, a_{g}$ are known constants denoting the sonic speeds in the liquid and in the gas, respectively; $P_{l, 0}$ and $\rho_{l, 0}$ are the reference pressure and density given as constants; $\alpha_{l}, \alpha_{g} \in[0,1]$ denote the liquid and gas volume fractions, satisfying $\alpha_{l}+\alpha_{g}=1$. We note

(c) 2015 Yang et al. This article is distributed under the terms of the Creative Commons Attribution 4.0 International License (http://creativecommons.org/licenses/by/4.0/), which permits unrestricted use, distribution, and reproduction in any medium, provided you give appropriate credit to the original author(s) and the source, provide a link to the Creative Commons license, and indicate if changes were made. 
from the expression of $\rho_{l}$ and $\rho_{g}$ that the pressure satisfies

$$
P(m, n)=C^{0}\left(-b(m, n)+\sqrt{b(m, n)^{2}+c(m, n)}\right)
$$

with $C^{0}=\frac{1}{2} a_{l}^{2}, k_{0}=\rho_{l, 0}-\frac{P_{l, 0}}{a_{l}^{2}}>0, a_{0}=\left(\frac{a_{g}}{a_{l}}\right)^{2}$, and

$$
b(m, n)=k_{0}-m-\left(\frac{a_{g}}{a_{l}}\right)^{2} n=k_{0}-m-a_{0} n, \quad c(m, n)=4 k_{0}\left(\frac{a_{g}}{a_{l}}\right)^{2} n=4 k_{0} a_{0} n .
$$

For more information as regards the above models, we can refer to [1-4].

The investigation of the above models has been a topic during the last decade. There has been much progress on the numerical properties of this model or other relevant models recently. It was $[5,6]$ who initiated some work in this direction. For the one-dimensional case, when the liquid is incompressible and the gas is polytropic, the global existence and uniqueness of a weak solution to the free boundary value problem were studied in [69]. When both of the two fluids are compressible, for their results one can consult [10], where the existence of the global weak solution was obtained. Moreover, for the results as regards the $1 \mathrm{D}$ case of the two-flow model, more interesting phenomena are described, such as the unequal fluid velocity, the well-reservoir interaction by allowing two kinds of gas between well and formation (surrounding reservoir), external forces, and the general pressure law [11-13]. In [10], Evje and Karlsen gave the global existence of a weak solution Cauchy problem for model (1.1) in 1D. For a multi-dimensional result as regards the twofluid flow model, Yao et al. in [14] obtained the existence of the global weak solution to the $2 \mathrm{D}$ model when the initial energy is small and there is no initial vacuum. Later on, Wen et al. $[15,16]$ proved the blow-up criterion in terms of the upper bound of the liquid mass for a local strong solution to the 3D (or 2D) viscous liquid-gas two-phase flow model in the two different cases when there is an initial vacuum and no initial vacuum in a bounded domain. Yao et al. in [17] gave the blow-up criterion in terms of the $L^{1}\left(0, T ; L^{\infty}\right)$-norm of the velocity with Dirichlet boundary condition and Navier-slip boundary condition without the restriction on viscosity coefficients. In [18] the authors investigated the Cauchy problem of model (1.1) in the framework of Besov space and obtained the global existence and uniqueness of the strong solution for the initial data close to a stable equilibrium. For the non-conservative viscous compressible two-fluid system $(1.1)$, we can see $[5,19]$ for the global existence of weak solution. Guo et al. [20] have proved the global existence of strong solution to Cauchy problem of system (1.1), where the initial data may vanish in an open set. Recently, in [21] one proved the global existence of the classical solution, which is a continuation of [20].

In the present paper, we consider a three-dimensional viscous liquid-gas two-phase flow model in a half-space $\Omega=\left\{x \in \mathbb{R}^{3}: x_{3}>0\right\}$, and we obtain the global existence and uniqueness of classical solutions with vacuum subject to some smallness assumptions on the initial energy. Before this work, there have been some results on the global existence and uniqueness of strong and classical solutions with vacuum in $\mathbb{R}^{3}$. When the boundary conditions, such as the half-space problem, are involved, things will become more complicated. For the boundary problems, there is no clarity as to the boundary value of the effective viscous $u_{x}$ and vorticity so that some elliptic estimates are not easy to get. We extend the results from single-phase flow to two-phase flow. The technique in [22] and in some other references on liquid-gas two-phase flow plays a very important role in the proof. 
Before stating the main results, we explain the notations used throughout this paper. We will use the following conventions throughout this paper:

$$
\int f d x=\int_{\Omega} f d x
$$

We denote the standard homogeneous and inhomogeneous Sobolev spaces as follows:

$$
\begin{aligned}
& L^{r}=L^{r}(\Omega), \quad H^{k}=W^{k, 2}, \quad D^{k, r}=\left\{u \in L_{\mathrm{loc}}^{1}(\Omega) \mid\left\|\nabla^{k} u\right\|_{L^{r}}<\infty\right\}, \\
& \|u\|_{D^{k, r}}=\left\|\nabla^{k} u\right\|_{L^{r}}, \quad W^{k, r}=L^{r} \cap D^{k, r}, \quad D^{k}=D^{k, 2}, \\
& D^{1}=\left\{u \in L^{6} \mid\|\nabla u\|_{L^{2}}<\infty, \text { and } u=0 \text { as }|x| \rightarrow \infty\right\} .
\end{aligned}
$$

The potential energy function $G$ takes the following form:

$$
G\left(m, \frac{n}{m}\right)=m \int_{\tilde{m}}^{m} \frac{P\left(s, \frac{n}{m}\right)-P(\tilde{m}, \tilde{n})}{s^{2}} d s+\frac{m}{\tilde{m}} P(\tilde{m}, \tilde{n})-\frac{m}{\tilde{m}} P\left(\tilde{m}, \frac{n}{m} \tilde{m}\right),
$$

and the initial energy is given by

$$
C_{0}=\int\left(\frac{1}{2} m_{0}\left|u_{0}\right|^{2}+G\left(m_{0}, \frac{n_{0}}{m_{0}}\right)\right) d x
$$

Let

$$
\int\left|\nabla u_{0}\right|^{2} d x \leq M
$$

where $M$ is a positive constant. It follows that there is a $\delta_{1} \in(0,1]$ small enough, which will be fixed throughout, such that

$$
\tilde{m} \tilde{n} \geq \frac{2}{\delta_{1}\left(2+\delta_{1}\right)} .
$$

We define the convective derivative $\frac{D}{D t}$ by $\frac{D f}{D t}=\dot{f}=f_{t}+u \cdot \nabla f$.

Then we give the main result of this paper.

Theorem 1.1 Assume that the conditions (1.6)-(1.9) hold. For given positive constants $\bar{m}$, $\bar{n}, M$ (not necessarily small) with $\bar{m}>2 \tilde{m}$, the initial data $\left(m_{0}, n_{0}, u_{0}\right)$ satisfies

$$
\begin{aligned}
& 0 \leq \inf _{x} m_{0} \leq \sup _{x} m_{0} \leq \bar{m}, \quad 0 \leq \inf _{x} n_{0} \leq \sup _{x} n_{0} \leq \bar{n}, \\
& u_{0} \in D^{1} \cap D^{3}, \quad\left(m_{0}-\tilde{m}, n_{0}-\tilde{n}\right) \in H^{3},
\end{aligned}
$$

and the compatibility condition

$$
-\mu \Delta u_{0}-(\lambda+\mu) \nabla \operatorname{div} u_{0}+\nabla P\left(m_{0}, n_{0}\right)=m_{0} g
$$

for some $g \in D^{1}$ with $m_{0}^{\frac{1}{2}} g \in L^{2}$. Furthermore, assume that

$$
0 \leq \underline{s}_{0} m_{0} \leq n_{0} \leq \frac{\tilde{n}}{\tilde{m}} m_{0}
$$


where $\underline{s}_{0}$ is a positive constant, satisfying

$$
\frac{\tilde{n}}{2 \tilde{m}} \leq \underline{s}_{0} \leq \frac{\tilde{n}}{\tilde{m}}
$$

Then there exists a positive constant $\varepsilon$ depending on $\bar{m}, M, \tilde{m}, \tilde{n}, C^{0}, a_{0}, \underline{s}_{0}, \mu$, and $\lambda$, such that, if $C_{0} \leq \varepsilon$, then the Cauchy problem (1.1)-(1.3) has a unique global classical solution $(m, n, u)$ satisfying for any $0<\tau<T<\infty$,

$$
\begin{aligned}
& 0 \leq m \leq 2 \bar{m}, \quad 0 \leq \underline{s}_{0} m \leq n \leq \frac{\tilde{n}}{\tilde{m}} m, \quad x \in \Omega, \quad t \geq 0, \\
& (m-\tilde{m}, n-\tilde{n}) \in C\left(0, T ; H^{3}\right), \\
& u \in C\left(0, T ; D^{1} \cap D^{3}\right) \cap L^{2}\left(0, T ; D^{4}\right) \cap L^{\infty}\left(\tau, T ; D^{4}\right), \\
& \left(m_{t}, n_{t}\right) \in C\left(0, T ; H^{2}\right), \quad \sqrt{m} u_{t} \in L^{\infty}\left(0, T ; L^{2}\right), \quad \sqrt{m} u_{t t} \in L^{2}\left(0, T ; L^{2}\right), \\
& u_{t} \in L^{\infty}\left(0, T ; D^{1}\right) \cap L^{2}\left(0, T ; D_{2}\right) \cap L^{\infty}\left(\tau, T ; D^{2}\right) \cap H^{1}\left(\tau, T ; D^{1}\right) .
\end{aligned}
$$

We should mention that the ideas introduced by Wen et al. in [15], in Yao et al. in [14, 16], Yao et al. in [20, 21] for the two-fluid flow model, Cho and Kim in [23], Huang et al. in [24], Duan in [22], Hoff in [25], and Perepelitsa in [26] for the single-fluid Navier-Stokes equations play crucial roles in our proof here.

\section{A priori estimates}

Firstly, using similar methods to [27] and the references therein, we can obtain the local existence and the uniqueness of the solutions to (1.1)-(1.3) with the regularities as in Theorem 1.1. We omit it here for brevity. In the following of this section, we derive some $a$ priori estimates for a local classical solution of problem (1.1)-(1.3) on $\Omega \times[0, T]$ for some $T>0$, with the initial data $\left(m_{0}, n_{0}, u_{0}\right)$ satisfying (1.10)-(1.14).

Next, we give the well-known Gagliardo-Nirenberg inequality, which will be used frequently later.

Lemma 2.1 (Gagliardo-Nirenberg inequality) [14] For any $p, b^{\prime}, r^{\prime} \in[1, \infty]$ and any integer $l$ and $j$, there exist some generic constant $\alpha \in[0,1]$ and $C>0$, for every function $u \in C_{0}^{\infty}$, such that

$$
\left\|\nabla^{j} u\right\|_{L^{p}\left(\mathbb{R}^{N}\right)} \leq C\left\|\nabla^{l} u\right\|_{L^{b^{\prime}\left(\mathbb{R}^{N}\right)}}^{\alpha}\|u\|_{L^{r^{\prime}\left(\mathbb{R}^{N}\right)}}^{1-\alpha}
$$

where

$$
\frac{1}{p}=\frac{j}{N}+\alpha\left(\frac{1}{b^{\prime}}-\frac{l}{N}\right)+(1-\alpha) \frac{1}{r^{\prime}}, \quad \frac{j}{l} \leq \alpha \leq 1 .
$$

$\alpha \neq 1$, when $l-\frac{N}{b^{\prime}}=j$ and $1<p<\infty$.

Next, the following Zlotnik inequality will be used to get the uniform (in time) upper bound of the $m$ and $n$. 
Lemma 2.2 ([28]) Let the function y satisfy

$$
y^{\prime}(t)=g(y)+b^{\prime}(t), \quad \text { on }[0, T], \quad y(0)=y^{0},
$$

with $g \in C(\mathbb{R})$ and $y, b \in W^{1,1}(0, T)$. If $g(\infty)=-\infty$ and

$$
b\left(t_{2}\right)-b\left(t_{1}\right) \leq N_{0}+N_{1}\left(t_{2}-t_{1}\right)
$$

for all $0 \leq t_{1}<t_{2} \leq T$ with some $N_{0} \geq 0$ and $N_{1} \geq 0$, then

$$
y(t) \leq \max \left\{y^{0}, \bar{\zeta}\right\}+N_{0}<\infty, \quad \text { on }[0, T],
$$

where $\bar{\zeta}$ is a constant such that

$$
g(\zeta) \leq-N_{1} \quad \text { for } \zeta \geq \bar{\zeta}
$$

Lemma 2.3 ([29]) Assume $X \subset E \subset Y$ are Banach spaces and $X \hookrightarrow \hookrightarrow E$. Then the following embeddings are compact:
(i) $\quad\left\{\varphi: \varphi \in L^{q}(0, T ; X), \frac{\partial \varphi}{\partial t} \in L^{1}(0, T ; Y)\right\} \hookrightarrow \hookrightarrow L^{q}(0, T ; E), \quad$ if $1 \leq q \leq \infty$;
(ii) $\left\{\varphi: \varphi \in L^{\infty}(0, T ; X), \frac{\partial \varphi}{\partial t} \in L^{r}(0, T ; Y)\right\} \hookrightarrow \hookrightarrow C([0, T] ; E), \quad$ if $1<r \leq \infty$.

Firstly, we denote

$$
\begin{aligned}
& A_{1}(T)=\sup _{0<t \leq T} \sigma \int|\nabla u|^{2} d x+\int_{0}^{T} \int \sigma m|\dot{u}|^{2} d x d s, \\
& A_{2}(T)=\sup _{0<t \leq T} \sigma^{3} \int m|\dot{u}|^{2} d x+\int_{0}^{T} \int \sigma^{3}|\nabla \dot{u}|^{2} d x d s,
\end{aligned}
$$

here $\sigma(t)=\min \{1, t\}$. For any $(x, t) \in \Omega \times[0, T]$, we make the following a priori assumptions:

$$
0 \leq m(x, t) \leq 2 \bar{m}
$$

and

$$
A_{1}(T)+A_{2}(T) \leq 2 C_{0}^{\frac{1}{2}}
$$

Under the assumption (2.5), we make the following remark.

Remark 2.1 Under the conditions of Theorem 1.1, for any $0 \leq t \leq T$, we have

$$
0 \leq \underline{s}_{0} m \leq n \leq \frac{\tilde{n}}{\tilde{m}} m, \quad x \in \Omega .
$$


Proof In fact, define the particle trajectories $x=X(t, y)$ given by

$$
\left\{\begin{array}{l}
\frac{d}{d t} X(t, y)=u(X(t, y), t), \\
X(0, y)=y .
\end{array}\right.
$$

From $(1.1)_{1}$ and $(1.1)_{2}$, we have

$$
\left(\frac{n}{m}\right)_{t}+u \cdot \nabla\left(\frac{n}{m}\right)=0
$$

which implies

$$
\frac{n(x, t)}{m(x, t)}=\frac{n_{0}}{m_{0}}\left(X^{-1}(t, x)\right):=s_{0}=s_{0}(x, t)
$$

where $X^{-1}$ denotes the inverse of $X$. We obtain (2.7) together with (1.13).

In the following, $C$ denotes a generic constant depending only on $\bar{m}, \tilde{m}, \tilde{n}, a_{0}, C^{0}, \underline{s}_{0}, \mu$, $\lambda$, and the initial data may vary in different estimates; we write $C(\alpha)$ to emphasize that $C$ depends on $\alpha$.

Remark 2.2 Under the conditions of Theorem 1.1, we have

$$
\begin{aligned}
& 0 \leq P_{m} \leq C\left(C^{0}\right), \quad \text { in } \Omega \times[0, T], \\
& 2 a_{0} C^{0} \leq P_{n} \leq C\left(C^{0}, a_{0}, \underline{s}_{0}\right), \quad \text { in } \Omega \times[0, T]
\end{aligned}
$$

The above remark plays a key role in the proof of the upper bound of the density $(m, n)$. Now, the standard energy estimate is given as follows.

Proposition 2.1 If $(m, n, u)(x, t)$ is a classical solution of $(1.1)-(1.3)$ in $[0, T]$, satisfying the assumptions (2.5)-(2.6), then we have

$$
\begin{aligned}
& \sup _{0 \leq t \leq T} \int\left(\frac{1}{2} m|u|^{2}+G(m, n)\right) d x+\int_{0}^{T} \int\left(\mu|\nabla u|^{2}+(\lambda+\mu)|\operatorname{div} u|^{2}\right) d x d t \\
& \quad+\beta^{-1} \int_{0}^{T} \int_{\partial \Omega}|u|^{2} d S_{x} d t \leq C_{0} .
\end{aligned}
$$

Proof In [14, 20], one obtained this standard energy estimate in $\mathbb{R}^{N}(N=2,3)$. Using a similar argument to $[14,20]$, we can easily obtain this energy estimate and omit the details.

Notice that the function $G(m, n)$ is equivalent to $(m-\tilde{m})^{2}+(n-\tilde{n})^{2}$, which implies

$$
\begin{aligned}
& \sup _{0 \leq t \leq T} \int\left(m|u|^{2}+(m-\tilde{m})^{2}+(n-\tilde{n})^{2}\right) d x \\
& \quad+\int_{0}^{T} \int|\nabla u|^{2} d x d t+\beta^{-1} \int_{0}^{T} \int_{\partial \Omega}|u|^{2} d S_{x} d t \leq C C_{0} .
\end{aligned}
$$

Now, we use $\omega$ and $F$, denoting the vorticity matrix and the effective viscous flux, defined in the following form: 


$$
F \triangleq(2 \mu+\lambda) \operatorname{div} u-P(m, n)+P(\tilde{m}, \tilde{n}), \quad \omega \triangleq \nabla \times u,
$$

then we can rewrite $(1.1)_{3}$ in the form

$$
m \dot{u}^{j}=\partial_{j} F+\mu \partial_{k} \omega^{j, k}
$$

which implies

$$
\Delta F=\operatorname{div}(m \dot{u}), \quad \mu \Delta \omega^{j, k}=\partial_{k}\left(m \dot{u}^{j}\right)-\partial_{j}\left(m \dot{u}^{k}\right)
$$

and

$$
(\mu+\lambda) \Delta u^{j}=\partial_{j} F+(\mu+\lambda) \partial_{k} w^{j, k}+\partial_{j}(P(m, n)-P(\tilde{m}, \tilde{n})) .
$$

Together with the energy estimate, we can get the following lemma.

Lemma 2.4 If $(m, n, u)$ is a classical solution of (1.1)-(1.3), we have for any $p \in[2,6]$

$$
\begin{aligned}
& \|u\|_{L^{p}} \leq C(\bar{m}) C_{0}^{\frac{6-p}{4 p}}\|\nabla u\|_{L^{2}}^{\frac{3 p-6}{2 p}}+C_{0}^{\frac{6-p}{6 p}}\|\nabla u\|_{L^{2}} \\
& \|\nabla F\|_{L^{p}}+\|\nabla \omega\|_{L^{p}} \leq C\left(\|m \dot{u}\|_{L^{p}}+\|\nabla u\|_{L^{p}}\right) \\
& \|F\|_{L^{p}}+\|\omega\|_{L^{p}} \leq C\|m \dot{u}\|_{L^{2}}^{\frac{3 p-6}{2 p}}\left(\|\nabla u\|_{L^{2}}+\|P(m, n)-P(\tilde{m}, \tilde{n})\|_{L^{2}}\right)^{\frac{6-p}{2 p}} \\
& \quad+C\left(\|\nabla u\|_{L^{2}}+\|P(m, n)-P(\tilde{m}, \tilde{n})\|_{L^{2}}^{\frac{6-p}{2 p}}\|\nabla u\|_{L^{2}}^{\frac{3 p-6}{2 p}}\right) \\
& \|\nabla u\|_{L^{p}} \leq C\left(\|F\|_{L^{p}}+\|\omega\|_{L^{p}}\right)+C\|P(m, n)-P(\tilde{m}, \tilde{n})\|_{L^{p}} \\
& \|\nabla u\|_{L^{p}} \leq C\left(\|\nabla u\|_{L^{2}}^{\frac{6-p}{2 p}}\left(\|m \dot{u}\|_{L^{2}}+\|\nabla u\|_{L^{2}}\right)\right) \\
& \quad+C\left(\|P(m, n)-P(\tilde{m}, \tilde{n})\|_{L^{2}}+\|P(m, n)-P(\tilde{m}, \tilde{n})\|_{L^{6}}\right)^{\frac{3 p-6}{2 p}}
\end{aligned}
$$

Also, for $0 \leq t_{1} \leq t_{2} \leq T$, we have for any $p \geq 2$ and $r \geq 0$

$$
\int_{t_{1}}^{t_{2}} \int \sigma^{r}|P(m, n)-P(\tilde{m}, \tilde{n})|^{p} d x d s \leq C\left(\int_{t_{1}}^{t_{2}} \int \sigma^{r}|F|^{p} d x d s+C_{0}\right)
$$

Proof Using a similar argument to that in [22] (Lemma 4.3) and [30] (Lemma 3.3), we can obtain this lemma and omit the details.

The proofs of the following estimates are similar to [14, 24, 25, 30, 31].

Lemma 2.5 If $(m, n, u)$ is the classical solution of (1.1)-(1.3) in [0,T] as in Proposition 2.1, then we have

$$
\begin{aligned}
A_{1}(T) & =\sup _{0<t \leq T} \sigma \int|\nabla u|^{2} d x+\int_{0}^{T} \int \sigma m|\dot{u}|^{2} d x d t \\
& \leq C C_{0}+C \int_{0}^{T} \int \sigma|\nabla u|^{3} d x d t+C \int_{0}^{T} \int \sigma\left(|u|^{2}|\nabla u|+|u||\nabla u|^{2}\right) d x d t
\end{aligned}
$$


and

$$
\begin{aligned}
A_{2}(T)= & \sup _{0<t \leq T} \sigma^{3} \int m|\dot{u}|^{2} d x+\int_{0}^{T} \int \sigma^{3}|\nabla u|^{2} d x d t \\
\leq & C C_{0}+C A_{1}(T) \\
& +C \int_{0}^{T} \int \sigma^{3}\left[|u|^{4}+|\nabla u|^{4}+|\dot{u}||\nabla u||u|+|\dot{u}||\nabla u|^{2}\right] d x d t .
\end{aligned}
$$

Proof Multiplying $(1.1)_{3}$ by $\sigma \dot{u}$, and integrating over $\Omega$, we obtain

$$
\begin{aligned}
\int \sigma m|\dot{u}|^{2} d x & =\int(-\sigma \dot{u} \cdot \nabla P+\mu \sigma \Delta u \cdot \dot{u}+(\lambda+\mu) \sigma \nabla \operatorname{div} u \cdot \dot{u}) d x \\
& =\sum_{i=1}^{3} J_{i} .
\end{aligned}
$$

Integrating by parts and using $(1.1)_{1},(1.1)_{2}$, and the Cauchy inequality, we have

$$
\begin{aligned}
J_{1}= & \int-\sigma \dot{u} \cdot \nabla P d x \\
= & \left(\int \sigma \operatorname{div} u(P(m, n)-P(\tilde{m}, \tilde{n})) d x\right)_{t} \\
& -\int\left\{\sigma^{\prime} \operatorname{div} u(P(m, n)-P(\tilde{m}, \tilde{n}))+\sigma P(m, n)_{t} \operatorname{div} u+\sigma(u \cdot \nabla u) \cdot \nabla P\right\} d x \\
= & \left(\int \sigma \operatorname{div} u(P(m, n)-P(\tilde{m}, \tilde{n})) d x\right)_{t}-\int \sigma^{\prime} \operatorname{div} u(P(m, n)-P(\tilde{m}, \tilde{n})) d x \\
& +\int \sigma\left\{\left(m P_{m}+n P_{n}\right)(\operatorname{div} u)^{2}-P(m, n)(\operatorname{div} u)^{2}+P(m, n) \partial_{i} u^{j} \partial_{j} u^{i}\right\} d x \\
\leq & \left(\int \sigma \operatorname{div} u(P(m, n)-P(\tilde{m}, \tilde{n})) d x\right)_{t}+C\|\nabla u\|_{L^{2}}^{2}+C \sigma^{\prime} C_{0},
\end{aligned}
$$

and noting the boundary condition (2.13) and the outer normal vector $N=\left(N^{1}, N^{2}, N^{3}\right)=$ $(0,0,-1)$, we get

$$
\begin{aligned}
J_{2}= & \mu \sigma \int \Delta u \cdot \dot{u} d x \\
= & \mu \sigma \int \Delta u \cdot\left(u_{t}+u \cdot \nabla u\right) d x \\
= & -\frac{\mu}{2}\left(\int \sigma|\nabla u|^{2} d x\right)_{t}+\frac{\mu}{2} \int \sigma^{\prime}|\nabla u|^{2} d x-\mu \sigma \int \partial_{i} u^{j} \partial_{i} u^{k} \partial_{k} u^{j} d x \\
& +\frac{\mu}{2} \int \sigma \partial_{k} u^{k}\left(\partial_{i} u^{j}\right)^{2} d x+\mu \sigma \int_{\partial \Omega} \partial_{i} u^{j} \dot{u}^{j} N^{i} d S_{x} \\
= & -\frac{\mu}{2}\left(\int \sigma|\nabla u|^{2} d x\right)_{t}+\frac{\mu}{2} \int \sigma^{\prime}|\nabla u|^{2} d x-\mu \sigma \int \partial_{i} u^{j} \partial_{i} u^{k} \partial_{k} u^{j} d x \\
& +\frac{\mu}{2} \int \sigma \partial_{k} u^{k}\left(\partial_{i} u^{j}\right)^{2} d x-\frac{\mu}{2}\left(\sigma \int_{\partial \Omega} \beta^{-1}|u|^{2} d S_{x}\right)_{t} \\
& -\mu \sigma \int_{\partial \Omega} \beta^{-1} u^{j} u^{i} u_{i}^{j} d S_{x}+\frac{\mu}{2} \sigma^{\prime} \int_{\partial \Omega} \beta^{-1}|u|^{2} d S_{x}
\end{aligned}
$$




$$
\begin{aligned}
\triangleq & -\frac{\mu}{2}\left(\int \sigma|\nabla u|^{2} d x\right)_{t}+\frac{\mu}{2} \int \sigma^{\prime}|\nabla u|^{2} d x-\mu \sigma \int \partial_{i} u^{j} \partial_{i} u^{k} \partial_{k} u^{j} d x \\
& +\frac{\mu}{2} \int \sigma \partial_{k} u^{k}\left(\partial_{i} u^{j}\right)^{2} d x-\frac{\mu}{2}\left(\sigma \int_{\partial \Omega} \beta^{-1}|u|^{2} d S_{x}\right)_{t}+J_{2}^{1}+J_{2}^{2} .
\end{aligned}
$$

Now we need to estimate the boundary term $J_{2}^{1}$ and $J_{2}^{2}$. We apply the fact that for $h \in$ $\left(C^{1} \cap W^{1,1}\right)(\bar{\Omega})$,

$$
\int_{\partial \Omega} h(x) d S=\int_{\Omega \bigcap\left\{0 \leq x_{3} \leq 1\right\}}\left[h(x)+\left(x_{3}-1\right) h_{x_{3}}(x)\right] d x .
$$

Since $j, k \in\{1,2\}$, using the fact (2.29), and integrating by parts in the $x_{1}$ and $x_{2}$ directions imply

$$
\begin{aligned}
& J_{2}^{1}=-\mu \sigma \int_{\partial \Omega} \beta^{-1} u^{j} u^{i} u_{i}^{j} d S_{x} \\
& =-\mu \sigma \int_{\Omega \bigcap\left\{0 \leq x_{3} \leq 1\right\}} \beta^{-1}\left[u^{j} u^{i} u_{i}^{j}+\left(x_{3}-1\right)\left(u^{j} u^{i} u_{i}^{j}\right)_{x_{3}}\right] d x \\
& =-\mu \sigma \int_{\Omega \bigcap\left\{0 \leq x_{3} \leq 1\right\}} \beta^{-1}\left\{u^{j} u^{i} u_{i}^{j}+\left(x_{3}-1\right)\left(u_{x_{3}}^{j} u^{i} u_{i}^{j}+u^{j} u_{x_{3}}^{i} u_{i}^{j}\right)\right\} \\
& +\mu \sigma \int_{\Omega \bigcap\left\{0 \leq x_{3} \leq 1\right\}} \beta^{-1}\left(x_{3}-1\right)\left(u_{i}^{j} u^{i} u_{x_{3}}^{j}+u^{j} u_{i}^{i} u_{x_{3}}^{j}\right) d x \\
& \leq C \sigma \int_{\Omega}\left(|u|^{2}|\nabla u|+|u||\nabla u|^{2}\right) d x \\
& J_{2}^{2}=\frac{\mu}{2} \sigma^{\prime} \int_{\partial \Omega} \beta^{-1}|u|^{2} d S_{x} \\
& =\frac{\mu}{2} \sigma^{\prime} \int_{\Omega \bigcap\left\{0 \leq x_{3} \leq 1\right\}} \beta^{-1}\left[|u|^{2}+2\left(x_{3}-1\right) u \cdot u_{x_{3}}\right] d x \\
& \leq C \sigma^{\prime} \int|u|^{2} d x+C\|\nabla u\|_{L^{2}}^{2} \leq C \sigma^{\prime}\left(C_{0}+C_{0}^{\frac{2}{3}} \int|\nabla u|^{2} d x\right)+C\|\nabla u\|_{L^{2}}^{2} \\
& \leq C C_{0} \sigma^{\prime}+C\|\nabla u\|_{L^{2}}^{2}
\end{aligned}
$$

here we have used (2.18), then we can estimate $J_{2}$ as

$$
\begin{aligned}
J_{2} \leq & -\frac{\mu}{2}\left(\int \sigma|\nabla u|^{2} d x+\sigma \int_{\partial \Omega} \beta^{-1}|u|^{2} d S_{x}\right)_{t}+C\|\nabla u\|_{L^{2}}^{2} \\
& +C \sigma \int|\nabla u|^{3} d x+C \sigma \int_{\Omega}\left(|u|^{2}|\nabla u|+|u||\nabla u|^{2}\right) d x+C C_{0} \sigma^{\prime} .
\end{aligned}
$$

Similarly, we have

$$
\begin{aligned}
J_{3} & =(\lambda+\mu) \int \sigma \nabla(\operatorname{div} u) \cdot \dot{u} d x \\
& \leq-\frac{(\lambda+\mu)}{2}\left(\int \sigma|\operatorname{div} u|^{2} d x\right)_{t}+C\|\nabla u\|_{L^{2}}^{2}+\int \sigma|\nabla u|^{3} d x \\
& \leq-\frac{(\lambda+\mu)}{2}\left(\int \sigma|\operatorname{div} u|^{2} d x\right)_{t}+\int \sigma|\nabla u|^{3} d x+C\|\nabla u\|_{L^{2}}^{2} .
\end{aligned}
$$


Combing (2.28)-(2.33), then integrating the resulting inequality over $[0, T]$ and using Young's inequality, we can obtain

$$
\begin{aligned}
\sup _{0 \leq t \leq T} \sigma & \|\nabla u\|_{L^{2}}^{2}+\int_{0}^{T} \int \sigma m|\dot{u}|^{2} d x d t+\int_{0}^{T} \int \sigma|\operatorname{div} u|^{2} d x d t \\
& +\int_{0}^{T} \int_{\partial \Omega} \sigma \beta^{-1}|u|^{2} d S_{x} d t \\
\leq & C C_{0}+C \int_{0}^{T} \int \sigma|\nabla u|^{3} d x d t+C \int_{0}^{T} \int \sigma\left(|u|^{2}|\nabla u|+|\nabla u|^{2}|u|\right) d x d t
\end{aligned}
$$

Multiplying $\sigma^{3} \dot{u}^{j}\left(\frac{\partial}{\partial t}+\operatorname{div}(u \cdot)\right)$ to $(1.1)_{3}^{j}$, summing with respect to $j$, and integrating the resulting equation over $\Omega$, we have

$$
\begin{aligned}
\left(\frac{\sigma^{3}}{2} \int m|\dot{u}|^{2} d x\right)_{t}= & \frac{3}{2} \int \sigma^{2} \sigma_{t} m|\dot{u}|^{2} d x+\mu \sigma^{3} \int \dot{u}^{j}\left[\Delta u_{t}^{j}+\operatorname{div}\left(u \Delta u^{j}\right)\right] d x \\
& -\sigma^{3} \int \dot{u}^{j}\left[\partial_{j} P_{t}+\operatorname{div}\left(\partial_{j} P u\right)\right] d x \\
& +(\lambda+\mu) \sigma^{3} \int \dot{u}^{j}\left[\partial_{t} \partial_{j} \operatorname{div} u+\operatorname{div}\left(u \partial_{j} \operatorname{div} u\right)\right] d x=\sum_{i=1}^{4} H_{i}
\end{aligned}
$$

Integrating by parts and using Young's inequality again, we have

$$
\begin{aligned}
H_{2}= & \mu \int \sigma^{3} \dot{u}^{j}\left[\Delta u_{t}^{j}+\operatorname{div}\left(u \Delta u^{j}\right)\right] d x \\
= & -\mu \int \sigma^{3}\left[|\nabla \dot{u}|^{2}+\partial_{i} \dot{u}^{j} \partial_{k} u^{k} \partial_{i} u^{j}-\partial_{i} \dot{u}^{j} \partial_{i} u^{k} \partial_{k} u^{j}-\partial_{i} u^{j} \partial_{i} u^{k} \partial_{k} \dot{u}^{j}\right] d x \\
& -\mu \int_{\partial \Omega} \sigma^{3} \beta^{-1}|\dot{u}|^{2} d S_{x}+\mu \int_{\partial \Omega} \sigma^{3} \dot{u}^{j} u_{k}^{j} u^{k} d S_{x}-\mu \int_{\partial \Omega} \sigma^{3} \partial_{k} \dot{u}^{j} u^{k} \partial_{3} u^{j} d S_{x} \\
\leq & -\frac{\mu}{2} \int \sigma^{3}|\nabla \dot{u}|^{2} d x+C \int \sigma^{3}|\nabla u|^{4} d x-\mu \int_{\partial \Omega} \sigma^{3} \beta^{-1}|\dot{u}|^{2} d S_{x} \\
& +C \mu \int \sigma^{3}\left[|u||\nabla u||\dot{u}|+|u||\nabla u||\nabla \dot{u}|+|\nabla u|^{2}|\dot{u}|\right] d x \\
\leq & -\frac{\mu}{4} \int \sigma^{3}|\nabla \dot{u}|^{2} d x+C \int \sigma^{3}|\nabla u|^{4} d x-\mu \int_{\partial \Omega} \sigma^{3} \beta^{-1}|\dot{u}|^{2} d S_{x} \\
& +C \int \sigma^{3}\left(|\nabla u|^{4}+|u|^{4}\right) d x+C \mu \int \sigma^{3}\left[|u||\nabla u||\dot{u}|+|\nabla u|^{2}|\dot{u}|\right] d x,
\end{aligned}
$$

where we have used

$$
\int_{\partial \Omega} \sigma^{3} \beta^{-1} \dot{u}^{j} u_{k}^{j} u^{k} \partial_{3} u^{j} d S_{x} \leq C \int_{\Omega} \sigma^{3}|\nabla u||u||\nabla \dot{u}| d x
$$

and

$$
\int_{\partial \Omega} \sigma^{3} \partial_{k} \dot{u}^{j} u^{k} d S_{x} \leq C \int_{\Omega} \sigma^{3}\left[|u||\nabla u||\dot{u}|+|u||\nabla u||\nabla \dot{u}|+|\nabla u|^{2}|\dot{u}|\right] d x .
$$


From $(1.1)_{1}$ and $(1.1)_{2}$, we have

$$
\begin{aligned}
H_{3}= & -\int \sigma^{3} \dot{u}^{j}\left[\partial_{j} P_{t}+\operatorname{div}\left(\partial_{j} P u\right)\right] d x \\
= & -\int \sigma^{3}\left[P_{m}(m \operatorname{div} u+u \cdot \nabla m) \partial_{j} \dot{u}^{j}+P_{n}(n \operatorname{div} u+u \cdot \nabla n) \partial_{j} \dot{u}^{j}\right] d x \\
& -\int \sigma^{3} P(m, n) \partial_{j}\left(\partial_{k} \dot{u}^{j} u^{k}\right) d x \\
= & \int \sigma^{3}\left[-P_{m} m \operatorname{div} u \partial_{j} \dot{u}^{j}-P_{n} n \operatorname{div} u \partial_{j} \dot{u}^{j}+\partial_{k}\left(\partial_{j} \dot{u}^{j} u^{k}\right) P-P\left(\partial_{j}\left(\partial_{k} \dot{u}^{j} u^{k}\right)\right)\right] d x \\
= & \int \sigma^{3}\left[-P_{m} m \operatorname{div} u \partial_{j} \dot{u}^{j}-P_{n} n \operatorname{div} u \partial_{j} \dot{u}^{j}+\partial_{j} \dot{u}^{j} \operatorname{div} u P-\partial_{k} \dot{u}^{j} \partial_{j} u^{k} P\right] d x \\
\leq & C \int \sigma^{3}|\nabla u||\nabla \dot{u}| d x \leq \frac{\mu}{8} \int \sigma^{3}|\nabla \dot{u}|^{2} d x+C \int \sigma^{3}|\nabla u|^{2} d x .
\end{aligned}
$$

Similarly,

$$
H_{4} \leq-\frac{\lambda+\mu}{2} \int \sigma|\operatorname{div} \dot{u}|^{2} d x+C \int \sigma|\nabla u|^{4} d x
$$

Combining (2.35)-(2.40) and integrating the result inequality over $(0, T)$, noting that $\int_{0}^{T} \int \sigma^{\prime} m|\dot{u}|^{2} d x d t \leq \int m|\dot{u}|^{2} d x \leq A_{1}(T)$, we can obtain (2.25), thus we complete the proof of Lemma 2.5 .

The following lemma will be applied to bound the higher-order terms occurring on the right hand side of (2.24) and (2.25).

Lemma 2.6 If $(m, n, u)$ is the classical solution of (1.1)-(1.3) in [0,T] as in Proposition 2.1, then there is a positive constant $T_{1}$ such that

$$
\sup _{t \in\left[0, T_{1} \wedge T\right]} \int|\nabla u|^{2} d x+\int_{0}^{T_{1} \wedge T} \int m\left|\dot{u}^{2}\right| d x d t \leq C(1+M)
$$

Proof In fact, multiplying $(1.1)_{3}$ by $\dot{u}$, then integrating the resulting equality over $\Omega \times[0, t]$, we have

$$
\int_{0}^{t} \int m|\dot{u}|^{2} d x d s=\int_{0}^{t} \int\{-\dot{u} \cdot \nabla P(m, n)+\mu \Delta u \cdot u+(\mu+\lambda) \nabla(\operatorname{div} u) \cdot \dot{u}\} d x d s
$$

Using a similar argument to that in the proof of (2.24), we have

$$
\begin{aligned}
& \int|\nabla u|^{2} d x+\int_{0}^{t} \int m|\dot{u}|^{2} d x d s \\
& \leq C\left(C_{0}+M\right)+C \int_{0}^{t} \int|\nabla u|^{3} d x d s+\int_{0}^{t} \int|u|^{2}|\nabla u|+|u||\nabla u|^{2} d x d s .
\end{aligned}
$$

From (2.5), (2.6), (2.14), (2.19)-(2.23), using Young's inequality and the Hölder inequality, we obtain 


$$
\begin{aligned}
\int_{0}^{t} \int|\nabla u|^{3} d x d s \leq & C \int_{0}^{t} \int\left(|F|^{3}+|\omega|^{3}\right) d x d s+C \int_{0}^{t} \int[P(m, n)-P(\tilde{m}, \tilde{n})]^{3} d x d s \\
\leq & C \int_{0}^{t}\left(\|F\|_{L^{2}}^{\frac{3}{2}}\|\nabla F\|_{L^{2}}^{\frac{3}{2}}+\|\omega\|_{L^{2}}^{\frac{3}{2}}\|\nabla \omega\|_{L^{2}}^{\frac{3}{2}}\right) d s+C C_{0} \\
\leq & C \int_{0}^{t}\left(\|\nabla u\|_{L^{2}}+\|P(m, n)-P(\tilde{m}, \tilde{n})\|_{L^{2}}\right)^{\frac{3}{2}} \\
& \times\left(\|m \dot{u}\|_{L^{2}}+\|\nabla u\|_{L^{2}}\right)^{\frac{3}{2}} d s+C C_{0} \\
\leq & \frac{1}{4} \int_{0}^{t} \int m|\dot{u}|^{2} d x d s+C \int_{0}^{t}\|\nabla u\|_{L^{2}}^{6} d s+C C_{0}
\end{aligned}
$$

and

$$
\begin{aligned}
& \int_{0}^{t} \int|u|^{2}|\nabla u|+|u||\nabla u|^{2} d x d s \\
& \quad \leq C \int_{0}^{t} \int\left(|\nabla u|^{2}+|u|^{4}\right) d x d s+C \int_{0}^{t}\|\nabla u\|_{L^{3}}\|\nabla u\|_{L^{2}}^{2} d s \\
& \quad \leq C \int_{0}^{t}\left[C_{0}^{\frac{1}{2}}\|\nabla u\|_{L^{2}}^{3}+C_{0}^{\frac{1}{3}}\|\nabla u\|_{L^{2}}^{4}\right] d s+C \int_{0}^{t}\left(\|\nabla u\|_{L^{3}}^{3}+\|\nabla u\|_{L^{2}}^{3}\right) d s \\
& \quad \leq \frac{1}{4} \int_{0}^{t} \int m \dot{u} d x d s+C \int_{0}^{t}\left(\|\nabla u\|_{L^{2}}^{3}+\|\nabla u\|_{L^{2}}^{4}+\|\nabla u\|_{L^{2}}^{6}\right) d s+C C_{0},
\end{aligned}
$$

which implies

$$
\begin{aligned}
& \int|\nabla u|^{2} d x+\int_{0}^{t} \int m|\dot{u}|^{2} d x d s \\
& \leq C(1+M)+C t\left(1+\sup _{s \in[0, t]}\|\nabla u(\cdot, t)\|_{L^{2}}^{6}\right) \\
& \leq C(1+M)+C t \sup _{s \in[0, t]}\|\nabla u(\cdot, t)\|_{L^{2}}^{6} \leq C(1+M),
\end{aligned}
$$

we can easily obtain (2.41) when we choose $T_{1}=\min \left\{1, \frac{1}{8 C^{3}(1+M)^{2}}\right\}$.

Proposition 2.2 If $(m, n, u)$ is the classical solution of (1.1)-(1.3) in [0,T] as in Proposition 2.1, then there is a positive constant $C$ such that

$$
A_{1}(T)+A_{2}(T) \leq C_{0}^{\frac{1}{2}}
$$

provided $C_{0} \leq \varepsilon_{1}$.

Proof From Lemma 2.5, we get

$$
\begin{aligned}
A_{1}(T)+A_{2}(T) \leq & C C_{0}+C \int_{0}^{T} \int \sigma^{3}|\nabla u|^{4} d x d t+C \int_{0}^{T} \int \sigma|\nabla u|^{3} d x d t \\
& +C \int_{0}^{T} \sigma^{3}\|u\|_{L^{4}}^{4} d t+C \int_{0}^{T} \int \sigma^{3}\left[|u||\nabla u||\dot{u}|+|\nabla u|^{2}|\dot{u}|\right] d x d t \\
& +C \int_{0}^{T} \int \sigma\left(|\nabla u||u|^{2}+|\nabla u||u|\right) d x d t
\end{aligned}
$$


We now estimate the term of the right hand sides of (2.45). Using (2.21), we have

$$
\int_{0}^{T} \int \sigma^{3}|\nabla u|^{4} d x d t \leq C \int_{0}^{T} \int \sigma^{3}\left[|F|^{4}+|\omega|^{4}+|P(m, n)-P(\tilde{m}, \tilde{n})|^{4}\right] d x d t .
$$

From (2.5)-(2.6), (2.14), and (2.20)-(2.22), we have

$$
\begin{aligned}
\int_{0}^{T} & \int \sigma^{3}\left(|F|^{4}+|\omega|^{4}\right) d x d t \\
\leq & C \int_{0}^{T} \sigma^{3}\left(\|\nabla u\|_{L^{2}}+\|P(m, n)-P(\tilde{m}, \tilde{n})\|_{L^{2}}\right)\|m \dot{u}\|_{L^{2}}^{3} d t \\
& +C \int_{0}^{T} \sigma^{3}\|\nabla u\|_{L^{2}}^{4} d t+C \int_{0}^{T} \sigma^{3}\|P(m, n)-P(\tilde{m}, \tilde{n})\|_{L^{2}}\|\nabla u\|_{L^{2}}^{3} d t \\
\leq & C \sup _{0<t \leq T}\left[\sigma^{\frac{3}{2}}\|\sqrt{m} \dot{u}\|_{L^{2}}\left(\sigma^{\frac{1}{2}}\|\nabla u\|_{L^{2}}+C_{0}^{\frac{1}{2}}\right)\right] \int_{0}^{T} \int \sigma m|\dot{u}|^{2} d x d t \\
& +C C_{0}^{\frac{3}{2}} \sup _{0<t \leq T}\left(\sigma^{\frac{1}{2}}\|\nabla u\|_{L^{2}}\right)+C C_{0} \sup _{0<t \leq T}\left(\sigma\|\nabla u\|_{L^{2}}^{2}\right) \\
\leq & C\left(A_{1}^{\frac{1}{2}}(T)+C_{0}^{\frac{1}{2}}\right) A_{2}^{\frac{1}{2}}(T) A_{1}(T)+C C_{0}^{\frac{3}{2}} A_{1}(T)^{\frac{1}{2}}+C C_{0} A_{1}(T) \\
\leq & C C_{0},
\end{aligned}
$$

using (2.23) and (2.47), we get

$$
\int_{0}^{T} \int \sigma^{3}|P(m, n)-P(\tilde{m}, \tilde{n})|^{4} d s \leq C\left(\int_{0}^{T} \int \sigma^{3}|F|^{4} d x d s+C_{0}\right) \leq C C_{0} .
$$

Notice that

$$
\int_{0}^{T} \int \sigma|\nabla u|^{3} d x d s=\int_{0}^{T_{1} \wedge T} \int \sigma|\nabla u|^{3} d x d s+\int_{T_{1} \wedge T}^{T} \int \sigma|\nabla u|^{3} d x d s .
$$

By Young's inequality, (2.14) and (2.46)-(2.47), we obtain

$$
\begin{aligned}
\int_{T_{1} \wedge T}^{T} \int \sigma|\nabla u|^{3} d x d s \leq & \int_{T_{1} \wedge T}^{T} \int \sigma\left(|\nabla u|^{4}+|\nabla u|^{2}\right) d x d s \\
\leq & C \int_{T_{1} \wedge T}^{T} \int \sigma^{3}|\nabla u|^{4} d x d s \\
& +\int_{T_{1} \wedge T}^{T} \int|\nabla u|^{2} d x d s \leq C C_{0},
\end{aligned}
$$

and by (2.14), (2.22), and Lemma 2.6, we get

$$
\begin{aligned}
\int_{0}^{T_{1} \wedge T} \sigma\|\nabla u\|_{L^{3}}^{3} d t \leq & C \int_{0}^{T_{1} \wedge T} \sigma\|\nabla u\|_{L^{2}}^{\frac{3}{2}}\left(\|m \dot{u}\|_{L^{2}}^{\frac{3}{2}}+\|\nabla u\|_{L^{2}}^{\frac{3}{2}}+C_{0}^{\frac{1}{4}}+C_{0}^{\frac{3}{4}}\right) d t \\
\leq & C \int_{0}^{T_{1} \wedge T}\left(\sigma^{\frac{1}{4}}\|\nabla u\|_{L^{2}}^{\frac{3}{2}}\right)\left(\sigma \int m|\dot{u}|^{2} d x\right)^{\frac{3}{4}} d t \\
& +C \int_{0}^{T_{1} \wedge T} \sigma\|\nabla u\|_{L^{2}}^{3} d s+C C_{0}
\end{aligned}
$$




$$
\begin{aligned}
& \leq C \sup _{t \in\left(0, T_{1} \wedge T\right]}\left(\left(\sigma\|\nabla u\|_{L^{2}}^{2}\right)^{\frac{1}{4}}\|\nabla u\|_{L^{2}}^{\frac{1}{2}}\right) \\
& \quad \times \int_{0}^{T_{1} \wedge T}\|\nabla u\|_{L^{2}}^{\frac{1}{2}}\left(\sigma \int m|\dot{u}|^{2} d x\right)^{\frac{3}{4}} d t \\
& \quad+\sup _{t \in\left(0, T_{1} \wedge T\right]}\left(\sigma\|\nabla u\|_{L^{2}}^{2}\right)^{\frac{1}{2}} \int_{0}^{T_{1} \wedge T} \sigma^{\frac{1}{2}}\|\nabla u\|_{L^{2}}^{2} d t+C C_{0} \\
& \leq C A_{1}(T) C_{0}^{\frac{1}{4}}+C C_{0} \leq C C_{0}^{\frac{3}{4}} .
\end{aligned}
$$

Using (2.14), (2.18)-(2.22) we get

$$
\begin{aligned}
& \int_{0}^{T} \int \sigma^{3}|u|^{4} d x d t \leq C \int_{0}^{T} \sigma^{3}\left(C_{0}^{\frac{1}{2}}\|\nabla u\|_{L^{2}}^{3}+C_{0}^{\frac{1}{3}}\|\nabla u\|_{L^{2}}^{4}\right) d t \leq C C_{0}, \\
& \int_{0}^{T} \int \sigma\left(|\nabla u||u|^{2}+|\nabla u|^{2}|u|\right) d x \\
& \quad \leq C \int_{0}^{T} \int|\nabla u|^{2} d x d t+\int_{0}^{T} \int \sigma^{2}|u|^{4} d x d t+\int_{0}^{T} \sigma\|\nabla u\|_{L^{3}}\|\nabla u\|_{L^{2}}^{2} d t \\
& \quad \leq C \int_{0}^{T} \int|\nabla u|^{2} d x d t+\int_{0}^{T} \int \sigma^{2}|u|^{4} d x d t+C \int \sigma\left(\|\nabla u\|_{L^{3}}^{3}+\|\nabla u\|_{L^{2}}^{3}\right) d t \\
& \quad \leq C C_{0}^{\frac{3}{4}},
\end{aligned}
$$

and

$$
\begin{aligned}
& \int_{0}^{T} \int \sigma\left(\left.|u \| \nabla u| \dot{u}|+| \nabla u\right|^{2}|\dot{u}|\right) d x d t \\
& \leq \int_{0}^{T} \sigma^{3}\|u\|_{L^{3}}\|\nabla u\|_{L^{2}}\|\nabla \dot{u}\|_{L^{2}} d t+C \int_{0}^{T} \sigma^{3}\|\nabla u\|_{L^{3}}\|\nabla u\|_{L^{2}}\|\nabla \dot{u}\|_{L^{2}} d t \\
& \leq C \int_{0}^{T} \sigma^{3}\left[C_{0}^{\frac{1}{4}}\|\nabla u\|_{L^{2}}^{\frac{1}{2}}+C_{0}^{\frac{1}{6}}\|\nabla u\|_{L^{2}}\right]\|\nabla u\|_{L^{2}}\|\nabla \dot{u}\|_{L^{2}} d t \\
& \quad+C \int_{0}^{T} \sigma^{3}\|\nabla u\|_{L^{3}}\|\nabla u\|_{L^{2}}\|\nabla \dot{u}\|_{L^{2}} d t \\
& \leq C C_{0}^{\frac{1}{4}} \int_{0}^{T} \sigma^{3}\|\nabla u\|_{L^{2}}^{\frac{3}{2}}\|\nabla \dot{u}\|_{L^{2}} d t+C C_{0}^{\frac{1}{6}} \int_{0}^{T} \sigma^{3}\|\nabla u\|_{L^{2}}^{2}\|\nabla \dot{u}\|_{L^{2}} d t \\
& \quad+C \int_{0}^{T} \sigma^{3}\|\nabla u\|_{L^{3}}\|\nabla u\|_{L^{2}}\|\nabla \dot{u}\|_{L^{2}} d t \\
& \leq C C_{0}^{\frac{3}{4}}
\end{aligned}
$$

here we have used the following fact:

$$
\begin{aligned}
& \int_{0}^{T} \sigma^{3}\|\nabla u\|_{L^{3}}\|\nabla u\|_{L^{2}}\|\nabla \dot{u}\|_{L^{2}} d t \\
& \quad \leq C \int_{0}^{T} \sigma^{3}\|\nabla u\|_{L^{3}}^{3} d s+C \int_{0}^{T} \sigma^{3}\|\nabla u\|_{L^{2}}\|\nabla u\|_{L^{2}}^{\frac{1}{2}}\|\nabla \dot{u}\|_{L^{2}}^{\frac{3}{2}} d s \\
& \quad \leq C C_{0}^{\frac{3}{4}}+C A_{1}(T)^{\frac{1}{2}}\left(\int_{0}^{T} \sigma\|\nabla u\|_{L^{2}}^{2} d s\right)^{\frac{1}{4}}\left(\int_{0}^{T} \sigma^{3}\|\nabla \dot{u}\|_{L^{2}}^{2} d s\right)^{\frac{3}{4}} \leq C C_{0}^{\frac{3}{4}},
\end{aligned}
$$


which together with (2.45)-(2.54) give

$$
A_{1}(T)+A_{2}(T) \leq C C_{0}^{\frac{3}{4}} \leq C_{0}^{\frac{1}{2}},
$$

when we choose $\varepsilon_{1} \leq C(\bar{m}, M)^{-4}$. This completes the proof of Proposition 2.2.

From Proposition 2.2, we can obtain the following corollary.

Corollary 2.1 If $(m, n, u)$ is the classical solution of (1.1)-(1.3) in [0,T] as in Proposition 2.1, then there is a positive constant $C$ such that

$$
\begin{aligned}
& \sup _{0<t \leq T}\|\nabla u\|_{L^{2}}^{2}+\int_{0}^{T} \int m|\dot{u}|^{2} d x d t \leq C(\bar{m}, M), \\
& \sup _{0<t \leq T} \int \sigma m|\dot{u}|^{2} d x+\int_{0}^{T} \int \sigma|\nabla \dot{u}|^{2} d x d t \leq C(\bar{m}, M),
\end{aligned}
$$

provided $C_{0}<\varepsilon_{1}$.

Proof Obviously, we can get (2.57) by Lemma 2.6 and Proposition 2.2.

Next, we prove (2.58). Applying the operator $\sigma \dot{u}\left(\frac{\partial}{\partial t}+\operatorname{div}(u \cdot)\right)$ to $(1.1)_{3}$ and integrating the resulting equality over $\Omega \times[0, T]$ and by using integration by parts, $(1.1)_{1},(1.1)_{2}$, Young's inequality, (2.14), (2.22), and (2.57), we have

$$
\begin{aligned}
& \sup _{0<t \leq T} \int \sigma m|\dot{u}|^{2} d x+\int_{0}^{T} \int \sigma|\nabla \dot{u}|^{2} d x d t \\
& \leq \int_{0}^{T} \int \sigma_{t} m|\dot{u}|^{2} d x d t+C \int_{0}^{T} \int \sigma|\nabla u|^{4} d x d t+C(\bar{m}) C_{0} \\
& \quad+C \int_{0}^{T} \int \sigma|u|^{4} d x+C \int_{0}^{T} \int \sigma\left[|u||\nabla u \| \dot{u}|+|\nabla u|^{2}|\dot{u}|\right] d x d t \\
& \leq \int_{0}^{\sigma(T)} \int m|\dot{u}|^{2} d x d t+C \int_{T_{1} \wedge T}^{T} \int \sigma|\nabla u|^{4} d x d t+C \int_{0}^{T_{1} \wedge T} \int \sigma|\nabla u|^{4} d x d t \\
& \quad+C \int_{0}^{T} \int \sigma^{3}|u|^{4} d x d t+C \int_{0}^{T} \int \sigma\left[|u \| \nabla u||\dot{u}|+|\nabla u|^{2}|\dot{u}|\right] d x d t+C(\bar{m}) C_{0} \\
& \leq C(\bar{m}, M)+C \int_{T_{1} \wedge T}^{T} \int \sigma^{3}|\nabla u|^{4} d x d t+C \int_{0}^{T_{1} \wedge T} \int \sigma|\nabla u|^{4} d x d t \\
&+C \int_{0}^{T} \int \sigma\left[|u||\nabla u \| \dot{u}|+|\nabla u|^{2}|\dot{u}|\right] d x d t \\
& \leq C(\bar{m}, M)+C \int_{0}^{T_{1} \wedge T} \sigma\|\nabla u\|_{L^{2}}\left(\|m \dot{u}\|_{L^{2}}^{3}+\|P(m, n)-P(\tilde{m}, \tilde{n})\|_{L^{6}}^{3}\right. \\
&\left.+\|\nabla u\|_{L^{2}}^{3}+\|P(m, n)-P(\tilde{m}, \tilde{n})\|_{L^{2}}^{3}\right) d t \\
&+C \int_{0}^{T} \int \sigma\left[|u\|\nabla u\| \dot{u}|+|\nabla u|^{2}|\dot{u}|\right] d x d t \\
& \leq C(\bar{m}, M)+C(\bar{m}) \sup _{t \in\left(0, T_{1} \wedge T\right]}\left[\left(\sigma^{\frac{1}{2}}\|\nabla u\|_{L^{2}}\right)\left(\sigma^{\frac{1}{2}}\|m \dot{u}\|_{L^{2}}\right)\right]
\end{aligned}
$$




$$
\begin{aligned}
& \times \int_{0}^{T_{1} \wedge T}\|m \dot{u}\|_{L^{2}}^{2} d t+\sup _{t \in(0, T)}\left(\sigma\|\nabla u\|_{L^{2}}^{2}\right) \int_{0}^{T_{1} \wedge T}\|\nabla u\|_{L^{2}}^{2} d t+\frac{1}{2} \int_{0}^{T} \sigma\|\nabla \dot{u}\|_{L^{2}}^{2} d t \\
\leq & C(\bar{m}, M)+C(\bar{m}, M) \sup _{t \in(0, T]} \sigma^{\frac{1}{2}}\|m \dot{u}\|_{L^{2}}+\frac{1}{2} \int_{0}^{T} \sigma\|\nabla \dot{u}\|_{L^{2}}^{2} d t .
\end{aligned}
$$

This completes the proof of Corollary 2.1.

Next, we give the time-independent upper bound of $m$ and $n$ by using similar arguments to $[22,24]$. It is noted that $(2.11)$ plays a key role here.

Proposition 2.3 If $(m, n, u)$ is the classical solution of (1.1)-(1.3) in $[0, T]$ as in Proposition 2.1, then there is a positive constant $C$ such that

$$
\sup _{0 \leq t \leq T}\|m(t)\|_{L^{\infty}} \leq \frac{7 \bar{m}}{4}, \quad \sup _{0 \leq t \leq T}\|n(t)\|_{L^{\infty}} \leq \frac{7 \bar{m}}{4} \frac{\tilde{n}}{\tilde{m}}, \quad(x, t) \in \Omega \times[0, T]
$$

provided that $C_{0} \leq \varepsilon$.

Proof Rewrite the equation of the mass conservation $(1.1)_{1}$ as

$$
D_{t} m=g(m)+b^{\prime}(t)
$$

where

$$
\begin{aligned}
& D_{t} m \triangleq m_{t}+u \cdot \nabla m, \quad g(m) \triangleq-\frac{m}{2 \mu+\lambda}(P(m, n)-P(\tilde{m}, \tilde{n})), \\
& b(t) \triangleq-\frac{1}{2 \mu+\lambda} \int_{0}^{t} m F d t .
\end{aligned}
$$

For $t \in[0, \sigma(T)]$, by Lemma 2.1, (2.14), (2.19), and (2.20), we have, for all $0 \leq t_{1}<t_{2} \leq$ $\sigma(T)$,

$$
\begin{aligned}
\left|b\left(t_{2}\right)-b\left(t_{1}\right)\right| & \\
\leq & C(\bar{m}) \int_{t_{1}}^{t_{2}}\|F(\cdot, t)\|_{L^{\infty}} d t \\
\leq & C(\bar{m}) \int_{0}^{\sigma(T)}\|F(\cdot, t)\|_{L^{2}}^{1 / 4}\|\nabla F(\cdot, t)\|_{L^{6}}^{3 / 4} d t \\
\leq & C(\bar{m}) \int_{0}^{\sigma(T)}\left(\|\nabla u\|_{L^{2}}^{\frac{1}{4}}+\|P(m, n)-P(\tilde{m}, \tilde{n})\|_{L^{2}}^{\frac{1}{4}}\right) \\
& \times\left(\|\nabla \dot{u}\|_{L^{2}}^{\frac{3}{4}}+\|m \dot{u}\|_{L^{2}}^{\frac{3}{4}}+\|\nabla u\|_{L^{2}}^{\frac{3}{4}}+\|P(m, n)-P(\tilde{m}, \tilde{n})\|_{L^{2}}^{\frac{3}{4}}\right. \\
& \left.+\|P(m, n)-P(\tilde{m}, \tilde{n})\|_{L^{6}}^{\frac{3}{4}}\right) d t \\
\leq & C(\bar{m}) \int_{0}^{\sigma(T)}\left(\sigma^{-\frac{1}{2}}\left(\sigma^{\frac{1}{2}}\|\nabla u\|_{L^{2}}\right)^{\frac{1}{4}}+C_{0}^{\frac{1}{8}} \sigma^{-\frac{3}{8}}\right)\left[\left(\sigma\|\nabla \dot{u}\|_{L^{2}}^{2}\right)^{\frac{3}{8}}+\left(\left(\sigma\|m \dot{u}\|_{L^{2}}^{2}\right)^{\frac{3}{8}}\right)\right] d t \\
& +C(\bar{m}) \int_{0}^{\sigma(T)}\left(\sigma^{\frac{1}{2}}\|\nabla u\|_{L^{2}}\right)^{\frac{1}{4}} \sigma^{-\frac{1}{8}}\left(\|P(m, n)-P(\tilde{m}, \tilde{n})\|_{L^{2}}^{2}\right)^{\frac{3}{8}} d t+C(\bar{m}) C_{0}^{\frac{1}{2}}
\end{aligned}
$$




$$
\begin{aligned}
\leq & C(\bar{m}) C_{0}^{\frac{1}{16}} \int_{0}^{\sigma(T)}\left(\sigma^{-\frac{1}{2}}+1\right)\left[\left(\sigma\|\nabla \dot{u}\|_{L^{2}}^{2}\right)^{\frac{3}{8}}+\left(\sigma\|m \dot{u}\|_{L^{2}}^{2}\right)^{\frac{3}{8}}\right] d t \\
& +C(\bar{m}) C_{0}^{\frac{1}{16}} \int_{0}^{\sigma(T)} \sigma^{-\frac{1}{8}}\left(\|P(m, n)-P(\tilde{m}, \tilde{n})\|_{L^{2}}^{2}\right)^{\frac{3}{8}} d t+C(\tilde{m}) C_{0}^{\frac{1}{2}} \\
\leq & C(\bar{m}) C_{0}^{\frac{1}{16}}\left(1+\int_{0}^{1} \sigma^{-\frac{4}{5}} d t\right)^{\frac{5}{8}}\left[\left(\int_{0}^{\sigma(T)} \sigma\|\nabla \dot{u}\|_{L^{2}}^{2} d t\right)^{\frac{3}{8}}+\left(\int_{0}^{\sigma(T)} \sigma\|m \dot{u}\|_{L^{2}}^{2} d t\right)^{\frac{3}{8}}\right] \\
& +C(\bar{m}) C_{0}^{\frac{1}{16}}\left(\int_{0}^{\sigma(T)} \sigma^{-\frac{1}{5}} d t\right)^{\frac{5}{8}}\left(\int_{0}^{\sigma(T)}\|P(m, n)-P(\tilde{m}, \tilde{n})\|_{L^{2}}^{2} d t\right)^{\frac{3}{8}}+C(\tilde{m}) C_{0}^{\frac{1}{2}} \\
\leq & C(\bar{m}, M) C_{0}^{\frac{1}{16}}
\end{aligned}
$$

provided that $C_{0} \leq \varepsilon_{1}$. Therefore, for $t \in[0, \sigma(T)]$, choose $N_{1}=0$ and $N_{0}=C(\bar{m}, M) C_{0}^{\frac{1}{16}}$ and $\bar{\xi}=2 \tilde{m}$. Then

$$
\begin{aligned}
g(\xi) & =-\frac{\xi}{2 \mu+\lambda}\left(P\left(\xi, \xi s_{0}\right)-P(\tilde{m}, \tilde{n})\right) \\
& =-\frac{\xi}{2 \mu+\lambda}\left(P\left(\xi, s_{0} \xi\right)-P(\xi, \tilde{n})+P(\xi, \tilde{n})-P(\tilde{m}, \tilde{n})\right) \\
& =-\frac{\xi}{2 \mu+\lambda}\left(P_{m}\left(\tilde{m}+\theta_{1}(\xi-\tilde{m}), \tilde{n}\right)(\xi-\tilde{m})+P_{n}\left(\xi, \tilde{n}+\left(s_{0} \xi-\tilde{n}\right) \theta_{2}\right)\left(s_{0} \xi-\tilde{n}\right)\right) \\
& \triangleq-\frac{1}{2 \mu+\lambda} z(\xi)
\end{aligned}
$$

where $\theta_{1}, \theta_{2} \in(0,1)$ are constants. From Remark 2.2 and (1.14), we obtain

$$
z(\xi) \geq 2 a_{0} C^{0} \xi\left(\underline{s}_{0} \xi-\tilde{n}\right) \geq 4 a_{0} C^{0} \tilde{m}\left(2 \underline{s}_{0} \tilde{m}-\tilde{n}\right) \geq 0, \quad \text { for all } \xi \geq \bar{\xi}=2 \tilde{m},
$$

and Lemma 2.2 yields

$$
\sup _{t \in[0, \sigma(T)]}\|m\|_{L^{\infty}} \leq \max \{\bar{m}, 2 \tilde{m}\}+C(\bar{m}, M) C_{0}^{\frac{1}{16}} \leq \bar{m}+C(\bar{m}, M) C_{0}^{\frac{1}{16}} \leq \frac{3}{2} \bar{m}
$$

provided that

$$
C_{0} \leq \min \left\{\varepsilon_{1}, \varepsilon_{2}\right\}, \quad \text { where } \varepsilon_{2}=\left(\frac{\bar{m}}{2 C(\bar{m}, M)}\right)^{16} .
$$

When $t \in[\sigma(T), T]$, by using Lemma 2.1, Proposition 2.2, (2.14), (2.19), and (2.58), we have

$$
\begin{aligned}
\left|b\left(t_{2}\right)-b\left(t_{1}\right)\right| & \leq C(\bar{m}) \int_{t_{1}}^{t_{2}}\|F(\cdot, t)\|_{L^{\infty}} d t \\
& \leq \frac{a_{0} C^{0}}{2 \mu+\lambda}\left(t_{2}-t_{1}\right)+C(\bar{m}) \int_{t_{2}}^{t_{1}}\|F(\cdot, t)\|_{L^{\infty}}^{\frac{8}{3}} d t \\
& \leq \frac{a_{0} C^{0}}{2 \mu+\lambda}\left(t_{2}-t_{1}\right)+C(\bar{m}) \int_{t_{2}}^{t_{1}}\|F(\cdot, t)\|_{L^{2}}^{2 / 3}\|\nabla F(\cdot, t)\|_{L^{6}}^{2} d t \\
& \leq \frac{a_{0} C^{0}}{2 \mu+\lambda}\left(t_{2}-t_{1}\right)+C(\bar{m}) \int_{t_{2}}^{t_{1}}\left(\|\nabla \dot{u}\|_{L^{2}}^{\frac{2}{3}}+\|P(m, n)-P(\tilde{m}, \tilde{n})\|_{L^{2}}^{\frac{2}{3}}\right)
\end{aligned}
$$




$$
\begin{aligned}
& \times\left(\|m \dot{u}\|_{L^{6}}^{2}+\|\nabla u\|_{L^{2}}^{2}+\|P(m, n)-P(\tilde{m}, \tilde{n})\|_{L^{6}}^{2}\right) d t \\
\leq & \frac{a_{0} C^{0}}{2 \mu+\lambda}\left(t_{2}-t_{1}\right)+C(\bar{m}) C_{0}^{\frac{1}{6}} \int_{\sigma(T)}^{T}\|\nabla \dot{u}\|_{L^{2}}^{2} d t \\
& +C(\bar{m}) C_{0}^{\frac{1}{6}}+C(\bar{m}) C_{0}^{\frac{1}{2}}\left(t_{2}-t_{1}\right)+C_{0}^{\frac{1}{6}} \int_{\sigma(T)}^{T}\|m \dot{u}\|_{L^{2}}^{2} d t \\
\leq & \left(\frac{a_{0} C^{0}}{2 \mu+\lambda}+C(\bar{m}) C_{0}^{\frac{1}{2}}\right)\left(t_{2}-t_{1}\right)+C(\bar{m}) C_{0}^{\frac{2}{3}} \\
\leq & \frac{2 a_{0} C^{0}}{2 \mu+\lambda}\left(t_{2}-t_{1}\right)+C(\bar{m}) C_{0}^{\frac{2}{3}},
\end{aligned}
$$

provided that $C_{0} \leq\left\{\varepsilon_{1}, \varepsilon_{2}, \varepsilon_{3}\right\}$, where $\varepsilon_{3}=\left(\frac{a_{0} C^{0}}{C(\overline{\bar{m}})(2 \mu+\lambda)}\right)^{2}$. Therefore, for $t \in[\sigma(T), T]$, choose $N_{1}=\frac{2 a_{0} C^{0}}{2 \mu+\lambda}$ and $N_{0}=C(\bar{m}) C_{0}^{\frac{2}{3}}$ and $\bar{\xi}=\left(2+\delta_{1}\right) \tilde{m}$. From Remark 2.2, (1.9), and (1.14), we obtain

$$
\begin{aligned}
& z(\xi) \geq 2 a_{0} C^{0}\left(2+\delta_{1}\right) \tilde{m}\left(\left(2+\delta_{1}\right) \underline{s}_{0} \tilde{m}-\tilde{n}\right) \geq 2 a_{0} C^{0}\left(2+\delta_{1}\right) \tilde{m} \tilde{n} \frac{\delta_{1}}{2} \geq 2 a_{0} C^{0}, \\
& \quad \text { for all } \xi \geq \bar{\xi}=\left(2+\delta_{1}\right) \tilde{m},
\end{aligned}
$$

where $\delta_{1} \in(0,1]$ is a small constant. Then Lemma 2.3 yields

$$
\begin{aligned}
\sup _{t \in[\sigma(T), T]}\|m\|_{L^{\infty}} & \leq \max \left\{\frac{3}{2} \bar{m},\left(2+\delta_{1}\right) \tilde{m}\right\}+C\left(\bar{m}, \tilde{m}, \tilde{n}, C^{0}, a_{0}, \underline{s}_{0}, \mu, \lambda\right) C_{0}^{\frac{2}{3}} \\
& \leq \frac{3}{2} \bar{m}+C(\bar{m}) C_{0}^{\frac{2}{3}} \leq \frac{7}{4} \bar{m},
\end{aligned}
$$

provided that

$$
C_{0} \leq \varepsilon \triangleq \min \left\{\varepsilon_{1}, \varepsilon_{2}, \varepsilon_{3}, \varepsilon_{4}\right\}, \quad \text { where } \varepsilon_{4}=\left(\frac{\bar{m}}{4 C(\bar{m})}\right)^{\frac{3}{2}}
$$

Then (2.62) and (2.63) complete the proof of Proposition 2.3.

From now on, we will assume that the initial energy $C_{0}<\varepsilon$ and the constant $C$ may depend on $T,\|\sqrt{m} g\|_{L^{2}},\|\nabla g\|_{L^{2}},\left\|\left(m_{0}-\tilde{m}, n_{0}-\tilde{n}\right)\right\|_{H^{3}},\left\|u_{0}\right\|_{D^{1} \cap D^{3}}$, besides $\mu, \lambda, \tilde{m}, \tilde{n}, \bar{m}$, $a_{0}, C^{0}, \underline{s}_{0}$, and $M$, where $g$ is the same as in (1.12).

Finally, we give the proof of the high-order regularity estimates of $(m, n, u)$, which is due to $[22-24,30]$ for the single-fluid Navier-Stokes equations.

Proposition 2.4 ([20, 22]) If $(m, n, u)$ is a classical solution of $(1.1)-(1.3)$ in $[0, T]$, we have the following estimates:

$$
\begin{aligned}
& \sup _{0<t \leq T} \int m|\dot{u}|^{2} d x+\int_{0}^{T} \int|\nabla \dot{u}|^{2} d x d t \leq C, \\
& \sup _{0<t \leq T}\left(\|\nabla m\|_{L^{2} \cap L^{6}}+\|\nabla n\|_{L^{2} \cap L^{6}}+\|\nabla u\|_{H^{1}}\right)+\int_{0}^{T}\|\nabla u\|_{L^{\infty}} d t \leq C .
\end{aligned}
$$


Proof Applying the operator $\dot{u}\left(\frac{\partial}{\partial t}+\operatorname{div}(u \cdot)\right)$ to $(1.1)_{3}$ and integrating the resulting equality over $[0, T]$, we have

$$
\begin{aligned}
\left(\frac{1}{2} \int m|\dot{u}|^{2} d x\right)_{t}= & -\int \dot{u}^{j}\left[\partial_{j} P_{t}+\operatorname{div}\left(\partial_{j} P u\right)\right] d x+\int \mu \dot{u}^{j}\left(\Delta u_{t}^{j}+\operatorname{div}\left(u \Delta u^{j}\right)\right) d x \\
& +\int(\lambda+\mu) \dot{u}^{j}\left(\partial_{j} \partial_{t}(\operatorname{div} u)+\operatorname{div}\left(u \partial_{j}(\operatorname{div} u)\right)\right) d x .
\end{aligned}
$$

Using integration by parts, $(1.1)_{1},(1.1)_{2}$, and Young's inequality, we have

$$
\begin{aligned}
& \left(\int m|\dot{u}|^{2} d x\right)_{t}+\int|\nabla \dot{u}|^{2} d x \\
& \quad \leq C\left(\|\nabla u\|_{L^{4}}^{4}+\|\nabla u\|_{L^{2}}^{2}+\|u\|_{L^{4}}^{4}\right)+C \int\left(|u\|\nabla u\| \dot{u}|+|\nabla u|^{2}|\dot{u}|\right) d x \\
& \quad \leq C\|\nabla u\|_{L^{2}}\|\nabla u\|_{L^{6}}^{3}+C\|\nabla u\|_{L^{2}}^{3}+C \int\left(|u\|\nabla u\| \dot{u}|+|\nabla u|^{2}|\dot{u}|\right) d x+C \\
& \quad \leq C\left(\|F\|_{L^{6}}^{3}+\|\omega\|_{L^{6}}^{3}+\|P(m, n)-P(\tilde{m}, \tilde{n})\|_{L^{6}}^{3}\right)+\delta\|\nabla \dot{u}\|_{L^{2}}^{2}+C\|\nabla u\|_{L^{3}}^{3}+C \\
& \quad \leq C\left(\|\nabla F\|_{L^{2}}^{3}+\|\nabla \omega\|_{L^{2}}^{3}\right)+\frac{1}{2}\|\nabla \dot{u}\|_{L^{2}}^{2}+C\|\nabla u\|_{L^{3}}^{3}+C \\
& \quad \leq C\|\sqrt{m} \dot{u}\|_{L^{2}}^{4}+C\|\nabla u\|_{L^{3}}^{3}+\frac{1}{2}\|\nabla \dot{u}\|_{L^{2}}^{2}+C,
\end{aligned}
$$

using (2.22) and

$$
\begin{aligned}
\int_{0}^{T}\|\nabla u\|_{L^{3}}^{3} d s \leq & \int_{0}^{T \wedge T_{1}}\|\nabla u\|_{L^{3}}^{3} d s+\int_{T \wedge T_{1}}^{T} \sigma^{3}\|\nabla u\|_{L^{3}}^{3} d s \\
\leq & C+C \int_{0}^{T \wedge T_{1}}\|\nabla u\|_{L^{2}}^{\frac{3}{2}}\left(\|m \dot{u}\|_{L^{2}}^{\frac{3}{2}}+\|P(m, n)-P(\tilde{m}, \tilde{n})\|_{L^{2}}^{\frac{3}{2}}\right. \\
& \left.\times\|P(m, n)-P(\tilde{m}, \tilde{n})\|_{L^{6}}^{\frac{3}{2}}+\|\nabla u\|_{L^{2}}^{\frac{3}{2}}\right) d s \\
\leq & C
\end{aligned}
$$

and the compatibility condition, we can define

$$
\left.\sqrt{m} \dot{u}\right|_{t=0}=-\sqrt{m_{0}} g .
$$

Then Gronwall's inequality gives (2.64).

Next, we prove (2.65). For $p \in[2,6]$, differentiating $(1.1)_{1}$ with respect to $x_{i}$, and then multiplying both sides of the result equation by $p\left|\partial_{i} m\right|^{p-2} \partial_{i} m$, we get

$$
\begin{aligned}
& \left(|\nabla m|^{p}\right)_{t}+\operatorname{div}\left(|\nabla m|^{p} u\right)+(p-1)|\nabla m|^{p} \operatorname{div} u \\
& \quad+p|\nabla m|^{p-2}(\nabla m)^{T} \nabla u(\nabla m)+p m|\nabla m|^{p-2} \nabla m \cdot \nabla \operatorname{div} u=0 .
\end{aligned}
$$

Similarly, we can obtain

$$
\begin{aligned}
& \left(|\nabla n|^{p}\right)_{t}+\operatorname{div}\left(|\nabla n|^{p} u\right)+(p-1)|\nabla n|^{p} \operatorname{div} u \\
& \quad+p|\nabla n|^{p-2}(\nabla n)^{T} \nabla u(\nabla n)+p n|\nabla n|^{p-2} \nabla n \cdot \nabla \operatorname{div} u=0 .
\end{aligned}
$$


By the standard $L^{p}$-estimate for an elliptic system,

$$
\begin{aligned}
& -\mu \Delta u-(\lambda+\mu) \nabla \operatorname{div} u=m \dot{u}+\nabla P, \quad \text { in } \Omega, \\
& \left(u_{1}, u_{2}, u_{3}\right)=\beta\left(u_{x_{3}}^{1}, u_{x_{3}}^{2}, 0\right) \quad \text { in } \partial \Omega,
\end{aligned}
$$

we obtain

$$
\left\|\nabla^{2} u\right\|_{L^{p}} \leq C\left(\|m \dot{u}\|_{L^{p}}+\|\nabla P\|_{L^{p}}\right) .
$$

Now, we give the estimate for $\|\nabla u\|_{L^{\infty}}$, which is crucial to obtain the estimate of $\|(\nabla m, \nabla n)\|_{L^{p}}$. As in [22], we set $w=u-v ; w$ and $v$ satisfy

$$
\left\{\begin{array}{l}
-\mu \Delta v-(\mu+\lambda) \nabla \operatorname{div} v=-\nabla(P(m, n)-P(\tilde{m}, \tilde{n})), \quad \text { in } \Omega \\
\left(v^{1}(x), v^{2}(x), v^{3}(x)\right)=\beta\left(v_{x_{3}}^{1}(x), v_{x_{3}}^{2}(x), 0\right) \quad \text { on } \partial \Omega, t>0
\end{array}\right.
$$

then, by the standard regularity estimate for elliptic systems, we have

$$
\begin{aligned}
& \|\nabla v\|_{L^{q}} \leq C\left(\|P(m, n)-P(\tilde{m}, \tilde{n})\|_{L^{q}}\right), \\
& \left\|\nabla^{2} v\right\|_{L^{q}} \leq C\|\nabla(P(m, n)-P(\tilde{m}, \tilde{n}))\|_{L^{q}}, \quad \text { for } q \in[2, \infty)
\end{aligned}
$$

and $w$ satisfies

$$
\left\{\begin{array}{l}
-\mu \Delta w-(\mu+\lambda) \nabla \operatorname{div} w=m \dot{u}, \quad \text { in } \Omega, \\
\left(w^{1}(x), w^{2}(x), w^{3}(x)\right)=\beta\left(w_{x_{3}}^{1}(x), w_{x_{3}}^{2}(x), 0\right) \quad \text { on } \partial \Omega, t>0 .
\end{array}\right.
$$

Similarly,

$$
\left\|\nabla^{2} w\right\|_{L^{q}} \leq C\|m \dot{u}\|_{L^{q}}, \quad\|\nabla w\|_{L^{\infty}} \leq\left(\|m \cdot u\|_{L^{2}}+\|m \dot{u}\|_{L^{6}}\right) \quad \text { for } q \in(1, \infty) .
$$

In order to obtain $\|\nabla v\|_{L^{\infty}}$, we give the following fact.

Remark 2.3 Let $\Omega=\left\{x \in \mathbb{R}^{3} \mid x_{3}>0\right\}$ and $\nabla v \in W^{1, q}(\Omega)$ with $q \in(3, \infty)$. There exists a constant $C$ depending only on $q$ such that

$$
\|\nabla v\|_{L^{\infty}} \leq C\left(1+\ln \left(e+\left\|\nabla^{2} v\right\|_{L^{q}}\right)\right)\|\nabla v\|_{B M O}, \quad \text { with } q \in(3, \infty)
$$

here

$$
\begin{aligned}
& \|\nabla v\|_{B M O}=\|\nabla v\|_{L^{2}}+[\nabla v]_{B M O}, \quad[\nabla v]_{B M O}=\sup _{r>0, x \in \Omega} \frac{1}{\Omega_{r}(x)} \int_{\Omega_{r}(x)}\left|\nabla v(y)-\nabla v_{r}(x)\right| d y, \\
& \nabla v_{r}(x)=\frac{1}{\left|\Omega_{r}(x)\right|} \int_{\Omega_{r}(x)} \nabla v(y) d y .
\end{aligned}
$$

Then, by using the classical theory for elliptic systems, we have

$$
\|\nabla v\|_{B M O} \leq C\left(\|P(m, n)-P(\tilde{m}, \tilde{n})\|_{L^{2}}+\|P(m, n)-P(\tilde{m}, \tilde{n})\|_{L^{\infty}}\right) \leq C(\bar{m}),
$$


which together with (2.72) implies

$$
\|\nabla v\|_{L^{\infty}} \leq C\left(1+\ln \left(e+\left\|\nabla^{2} v\right\|_{L^{q}}\right)\right)
$$

From (2.66) and (2.67) we have

$$
\begin{aligned}
\partial_{t}\left(\|\nabla m\|_{L^{p}}+\|\nabla n\|_{L^{p}}\right) & \leq C\|\nabla u\|_{L^{\infty}}\left(\|\nabla m\|_{L^{p}}+\|\nabla n\|_{L^{p}}\right)+C\left\|\nabla^{2} u\right\|_{L^{p}} \\
& \leq C\left(1+\|\nabla u\|_{L^{\infty}}\right)\left(\|\nabla m\|_{L^{p}}+\|\nabla n\|_{L^{p}}\right)+C\|m \dot{u}\|_{L^{p}} .
\end{aligned}
$$

Then, using Remark 2.3 and (2.70), we obtain

$$
\begin{aligned}
\|\nabla u\|_{L^{\infty}} & \leq C\left(\|\nabla w\|_{L^{\infty}}+\|\nabla v\|_{L^{\infty}}\right) \\
& \leq C\|\nabla w\|_{L^{\infty}}+\left(1+\ln \left(e+\left\|\nabla^{2} v\right\|_{L^{q}}\right)\right) \\
& \leq C\left(1+\|m \dot{u}\|_{L^{6}}+\ln \left(e+\|\nabla m\|_{q}+\|\nabla n\|_{L^{q}}\right)\right) .
\end{aligned}
$$

We set

$$
f(t)=e+\|\nabla m\|_{L^{6}}+\|\nabla n\|_{L^{6}}, \quad g(t)=1+\|m \dot{u}\|_{L^{6}} .
$$

Substituting (2.74) into (2.75) with $p=6$, we have

$$
f^{\prime}(t) \leq C g(t) f(t)+C f(t) \ln f(t)+C g(t)
$$

which yields

$$
(\ln f(t))^{\prime} \leq C g(t)+C \ln f(t) .
$$

Then Lemma 2.4, (2.60), and (2.64) imply

$$
\int_{0}^{T} g(t) d t \leq C \int_{0}^{T}\left(1+\|m \dot{u}\|_{L^{6}}\right) d t \leq C \int_{0}^{T}\left(1+\|\nabla \dot{u}\|_{L^{2}}\right) d t \leq C
$$

and we get by using Gronwall's inequality and (2.76)

$$
\sup _{0 \leq t \leq T} f(t) \leq C,
$$

i.e.,

$$
\sup _{0 \leq t \leq T}\left(\|\nabla m\|_{L^{6}}+\|\nabla n\|_{L^{6}}\right) \leq C .
$$

From (2.75), (2.77), and (2.78), we obtain

$$
\int_{0}^{T}\|\nabla u\|_{L^{\infty}} d t \leq C .
$$


Setting $p=2$ in (2.74), we have

$$
\sup _{0 \leq t \leq T}\left(\|\nabla m\|_{L^{2}}+\|\nabla n\|_{L^{2}}\right) \leq C
$$

This completes the proof of Proposition 2.4.

Corollary 2.2 If $(m, n, u)$ is a classical solution of $(1.1)-(1.3)$ in $[0, T]$, we have the following estimates:

$$
\sup _{0 \leq t \leq T} \int m\left|u_{t}\right|^{2} d x+\int_{0}^{T} \int\left|\nabla u_{t}\right|^{2} d x d s \leq C .
$$

In order to get the higher-order regularity estimates of the solution, we need to bound $P_{m m}, P_{m n}, P_{n n}, P_{m m m}, P_{m m n}, P_{m n n}$, and $P_{n n n}$.

Lemma 2.7 Under the conditions of Theorem 1.1, we have

$$
\begin{aligned}
& \left|P_{m m}\right| \leq C, \quad\left|P_{m n}\right| \leq C, \quad\left|P_{n n}\right| \leq C, \\
& \left|P_{m m m}\right| \leq C, \quad\left|P_{m m n}\right| \leq C, \quad\left|P_{n n m}\right| \leq C, \\
& \left|P_{n n n}\right| \leq C, \quad \text { in } \mathbb{R}^{3} \times[0, T] .
\end{aligned}
$$

Proof Since

$$
\begin{aligned}
& P_{m m}=C^{0} \frac{c}{\left(b^{2}+c\right)^{\frac{3}{2}}}, \quad P_{n n}=-C^{0} \frac{4 k_{0} a_{0}^{2} m}{\left(b^{2}+c\right)^{\frac{3}{2}}}, \quad P_{m n}=C^{0} 2 k_{0} a_{0} \frac{\left(k_{0}-m\right)+a_{0} n}{\left(b^{2}+c\right)^{\frac{3}{2}}}, \\
& P_{m m n}=\frac{4 k_{0} a_{0} C^{0}}{\left(b^{2}+c\right)^{\frac{3}{2}}}-\frac{12 C^{0} k_{0} a_{0} n\left(k_{0} a_{0}+a_{0} m+a_{0}^{2} n\right)}{\left(b^{2}+c\right)^{\frac{5}{2}}}, \quad P_{m m m}=\frac{12 C^{0} k_{0} a_{0} n b}{\left(b^{2}+c\right)^{\frac{5}{2}}}, \\
& P_{n n n}=\frac{12 C^{0} k_{0} a_{0}^{2} m\left(k_{0} a_{0}+a_{0} m+a_{0}^{2} n\right)}{\left(b^{2}+c\right)^{\frac{5}{2}}}, \\
& P_{m n n}=\frac{2 k_{0} a_{0}^{2} C^{0}}{\left(b^{2}+c\right)^{\frac{3}{2}}}-\frac{6 C^{0} k_{0} a_{0}^{2}\left(k_{0}^{2}-m^{2}+2 a_{0} k_{0} n+a_{0}^{2} n\right)}{\left(b^{2}+c\right)^{\frac{5}{2}}},
\end{aligned}
$$

by (2.7) and (2.60), we can obtain (2.82) and (2.83) easily.

Proposition 2.5 If $(m, n, u)$ is a classical solution of (1.1)-(1.3) in $[0, T]$, we have the following estimates:

$$
\sup _{0 \leq t \leq T}\left(\|m-\tilde{m}\|_{H^{2}}+\|n-\tilde{n}\|_{H^{2}}+\|P(m, n)-P(\tilde{m}, \tilde{n})\|_{H^{2}}\right) \leq C
$$

Proof At first, we give the elliptic estimate as follows:

$$
\|\nabla u\|_{H^{2}} \leq C\left(\|F\|_{H^{2}}+\|\omega\|_{H^{2}}+\|P(m, n)-P(\tilde{m}, \tilde{n})\|_{H^{2}}\right)
$$


Then from $(1.1)_{1},(1.1)_{2}$, and the above estimate, we have

$$
\begin{aligned}
& \frac{d}{d t}\left(\left\|\nabla^{2} m\right\|_{L^{2}}^{2}+\left\|\nabla^{2} n\right\|_{L^{2}}^{2}\right) \\
& \quad \leq C\left(1+\|\nabla u\|_{L^{\infty}}\right)\left(\left\|\nabla^{2} m\right\|_{L^{2}}^{2}+\left\|\nabla^{2} n\right\|_{L^{2}}^{2}\right)+C\|\nabla u\|_{H^{2}}^{2}+C .
\end{aligned}
$$

Using Proposition 2.4 and the same idea as the proof in [22] (Lemma 4.3), we have

$$
\begin{aligned}
\|F\|_{H^{2}}+\|\omega\|_{H^{2}}+\|P(m, n)-P(\tilde{m}, \tilde{n})\|_{H^{2}} \\
\leq C\left(\|F\|_{H^{1}}+\|\omega\|_{H^{1}}+\|m \dot{u}\|_{H^{1}}+\|P(m, n)-P(\tilde{m}, \tilde{n})\|_{H^{1}}\right) \\
\quad+C\left(\left\|\nabla^{2} m\right\|_{L^{2}}\left\|\nabla^{2} n\right\|_{L^{2}}\right) \\
\leq C\left(1+\|m \dot{u}\|_{L^{2}}+\|\nabla(m \dot{u})\|_{L^{2}}\right)+C\left(\left\|\nabla^{2} m\right\|_{L^{2}}+\left\|\nabla^{2} n\right\|_{L^{2}}\right) \\
\leq C\left(1+\|\nabla \dot{u}\|_{L^{2}}+\|\nabla m\|_{L^{3}}\|\dot{u}\|_{L^{6}}\right)+C\left(\left\|\nabla^{2} m\right\|_{L^{2}}+\left\|\nabla^{2} n\right\|_{L^{2}}\right),
\end{aligned}
$$

which together with Proposition 2.4 and Gronwall's inequality yields

$$
\sup _{0 \leq t \leq T} \int\left(\left\|\nabla^{2} m\right\|_{L^{2}}+\left\|\nabla^{2} n\right\|_{L^{2}}\right) d x \leq C .
$$

From the lemma, we have $\sup _{0 \leq t \leq T}\|P(m, n)-P(\tilde{m}, \tilde{n})\|_{H^{2}} \leq C$. Thus we finish the proof of Proposition 2.5.

Proposition 2.6 If $(m, n, u)$ is a classical solution of (1.1)-(1.3) in $[0, T]$, we have the following estimates:

$$
\sup _{0 \leq t \leq T}\left(\left\|m_{t}\right\|_{H^{1}}+\left\|n_{t}\right\|_{H^{1}}+\left\|P_{t}\right\|_{H^{1}}\right)+\int_{0}^{T}\left(\left\|m_{t t}\right\|_{L^{2}}^{2}+\left\|n_{t t}\right\|_{L^{2}}^{2}+\left\|P_{t t}\right\|_{L^{2}}^{2}\right) d t \leq C .
$$

Proof From $(1.1)_{1},(2.1),(2.60)$ and (2.65), we get

$$
\left\|m_{t}\right\|_{L^{2}} \leq C\|u\|_{L^{\infty}}\|\nabla m\|_{L^{2}}+C\|\nabla u\|_{L^{2}} \leq C
$$

Similarly, we have

$$
\left\|n_{t}\right\|_{L^{2}} \leq C\|u\|_{L^{\infty}}\|\nabla n\|_{L^{2}}+C\|\nabla u\|_{L^{2}} \leq C .
$$

Applying the $\nabla$ operator to $(1.1)_{1}$ yields

$$
\partial_{j} m_{t}+\partial_{j} u^{i} \partial_{i} m+u^{i} \partial_{i} \partial_{j} m+\partial_{j} m \operatorname{div} u+m \partial_{j} \operatorname{div} u=0
$$

By (2.1), (2.60), (2.65), and (2.84), we can obtain

$$
\begin{aligned}
\left\|\nabla m_{t}\right\|_{L^{2}} & \leq C\|\nabla u\|_{L^{3}}\|\nabla m\|_{L^{6}}+C\|u\|_{L^{\infty}}\left\|\nabla^{2} m\right\|_{L^{2}}+\left\|\nabla^{2} u\right\|_{L^{2}} \\
& \leq C\|\nabla u\|_{L^{2}}^{\frac{1}{2}}\left\|\nabla^{2} u\right\|_{L^{2}}^{\frac{1}{2}}\left\|\nabla^{2} m\right\|_{L^{2}}+C\|u\|_{L^{2}}^{\frac{1}{4}}\left\|\nabla^{2} u\right\|_{L^{2}}^{\frac{3}{4}}+C\left\|\nabla^{2} u\right\|_{L^{2}} \leq C .
\end{aligned}
$$


Similarly, we have

$$
\left\|\nabla n_{t}\right\|_{L^{2}} \leq C
$$

Next, differentiating $(1.1)_{1}$ with respect to $t$ yields

$$
m_{t t}+u_{t} \cdot \nabla m+u \cdot \nabla m_{t}+m_{t} \operatorname{div} u+m \operatorname{div} u_{t}=0 .
$$

Thus, we get from Lemma (2.1), (2.65), (2.81), (2.84), (2.92), and (2.93)

$$
\begin{aligned}
\int_{0}^{T}\left\|m_{t t}\right\|_{L^{2}}^{2} d t & \leq C \int_{0}^{T}\left(\left\|u_{t}\right\|_{L^{6}}^{2}\|\nabla m\|_{L^{3}}^{2}+\left\|\nabla m_{t}\right\|_{L^{2}}^{2}+\left\|m_{t}\right\|_{L^{6}}^{2}\|\nabla u\|_{L^{3}}^{2}+\left\|\nabla u_{t}\right\|_{L^{2}}^{2}\right) d t \\
& \leq C .
\end{aligned}
$$

Similarly, we have

$$
\int_{0}^{T}\left\|\nabla n_{t t}\right\|_{L^{2}}^{2} d t \leq C
$$

The estimates for $P_{t}$ and $P_{t t}$ can be dealt with in a similar way.

Corollary $2.3[20,22]$ If $(m, n, u)$ is a classical solution of $(1.1)-(1.3)$ in $[0, T]$, we have the following estimates:

$$
\sup _{0 \leq t \leq T} \int\left|\nabla u_{t}\right|^{2} d x+\int_{0}^{T} \int m u_{t t}^{2} d x d t \leq C .
$$

Proposition 2.7 If $(m, n, u)$ is a classical solution of $(1.1)-(1.3)$ in $[0, T]$, we have the following estimates:

$$
\begin{aligned}
& \sup _{0 \leq t \leq T}\left(\|m-\tilde{m}\|_{H^{3}}+\|n-\tilde{n}\|_{H^{3}}+\|P(m, n)-P(\tilde{m}, \tilde{n})\|_{H^{3}}\right) \leq C, \\
& \sup _{0 \leq t \leq T}\|\nabla u\|_{H^{2}}+\int_{0}^{T}\left(\|\nabla u\|_{H^{3}}^{2}+\left\|\nabla u_{t}\right\|_{H^{1}}^{2}\right) d t \leq C .
\end{aligned}
$$

Proof By using the Hölder inequality, Young's inequality, Lemma 2.1, (2.60), (2.65), (2.84), and (2.87), we have

$$
\begin{aligned}
\|\nabla(m \dot{u})\|_{L^{2}}= & \left\|\nabla\left(m u_{t}+m u \cdot \nabla u\right)\right\|_{L^{2}} \leq C\left\|\left|\nabla m\left\|u_{t} \mid\right\|_{L^{2}}+C\left\|m \nabla u_{t}\right\|_{L^{2}}\right.\right. \\
& +C\||\nabla m\|u\| \nabla u|\|_{L^{2}}+C\left\|m|\nabla u|^{2}\right\|_{L^{2}}+C\left\|m \left|u\left\|\nabla^{2} u \mid\right\|_{L^{2}}\right.\right. \\
\leq & C\|\nabla m\|_{L^{3}}\left\|u_{t}\right\|_{L^{6}}+C\left\|\nabla u_{t}\right\|_{L^{2}}+C\|u\|_{L^{\infty}}\|\nabla m\|_{L^{3}}\|\nabla u\|_{L^{6}} \\
& +C\|\nabla u\|_{L^{3}}\|\nabla u\|_{L^{6}}+C\|u\|_{L^{\infty}}\left\|\nabla^{2} u\right\|_{L^{2}} \\
\leq & C,
\end{aligned}
$$

which together with (2.64) imply

$$
\sup _{0 \leq t \leq T}\|m \dot{u}\|_{H^{1}} \leq C
$$


The standard $H^{1}$-estimate for the elliptic equations gives

$$
\begin{aligned}
\left\|\nabla^{2} u\right\|_{H^{1}} & \leq C\|\mu \Delta u+(\mu+\lambda) \nabla \operatorname{div} u\|_{H^{1}} \\
& \leq C\|m \dot{u}+\nabla P\|_{H^{1}} \\
& \leq C\|m \dot{u}\|_{H^{1}}+C\|\nabla P\|_{H^{1}} \leq C,
\end{aligned}
$$

where we have used (1.1) $)_{3}$, Lemma 2.1, (2.60), (2.65), (2.84), and (2.87) give

$$
\sup _{0<t \leq T}\|\nabla u\|_{H^{2}} \leq C
$$

Next, by using the standard $L^{2}$-estimate for the elliptic system, we can obtain

$$
\begin{aligned}
\left\|\nabla^{2} u_{t}\right\|_{L^{2}} \leq & C\left\|\mu \Delta u_{t}+(\mu+\lambda) \nabla \operatorname{div} u_{t}\right\|_{L^{2}} \\
= & C\left\|m u_{t t}+m_{t} u_{t}+m_{t} u \cdot \nabla u+m u_{t} \cdot \nabla u+m u \cdot \nabla u_{t}+\nabla P_{t}\right\|_{L^{2}} \\
\leq & C\left\|m u_{t t}\right\|_{L^{2}}+C\left\|m_{t}\right\|_{L^{3}}\left\|u_{t}\right\|_{L^{6}}+C\|u\|_{L^{\infty}}\left\|m_{t}\right\|_{L^{3}}\|\nabla u\|_{L^{6}} \\
& +\left\|u_{t}\right\|_{L^{6}}\|\nabla u\|_{L^{3}}+C\|u\|_{L^{\infty}}\left\|\nabla u_{t}\right\|_{L^{2}}+C\left\|\nabla P_{t}\right\|_{L^{2}} \\
\leq & C\left\|m u_{t t}\right\|_{L^{2}}+C,
\end{aligned}
$$

which together with (2.96) imply

$$
\int_{0}^{T}\left\|\nabla u_{t}\right\|_{H^{1}}^{2} d t \leq C
$$

In order to estimate $\left\|\nabla^{2} u\right\|_{H^{2}}$, we use the standard $H^{2}$-estimate of the elliptic equations again to get

$$
\begin{aligned}
\left\|\nabla^{2} u\right\|_{H^{2}} & \leq C\|\mu \Delta u+(\mu+\lambda) \nabla \operatorname{div} u\|_{H^{2}} \\
& \leq C\|m \dot{u}\|_{H^{2}}+C\|\nabla P\|_{H^{2}} \\
& \leq C+C\left\|\nabla u_{t}\right\|_{H^{1}}+C\left(\left\|\nabla^{3} m\right\|_{L^{2}}+\left\|\nabla^{3} n\right\|_{L^{2}}\right),
\end{aligned}
$$

here we also have used (1.1) $)_{3}$, Lemma 2.1, Lemma 2.4, (2.101), and the following simple facts:

$$
\begin{aligned}
\left\|\nabla^{2}\left(m u_{t}\right)\right\|_{L^{2}} & \leq C\left\|\left|\nabla^{2} m\right|\left|u_{t}\right|\right\|_{L^{2}}+C\left\|\left|\nabla m\left\|\nabla u_{t} \mid\right\|_{L^{2}}+C\left\|\nabla^{2} u_{t}\right\|_{L^{2}}\right.\right. \\
& \leq C\left\|\nabla^{2} m\right\|_{L^{2}}\left\|\nabla u_{t}\right\|_{L^{2}}+C\|\nabla m\|_{L^{3}}\left\|\nabla u_{t}\right\|_{L^{6}}+C\left\|\nabla^{2} u\right\|_{L^{2}} \\
& \leq C+C\left\|\nabla u_{t}\right\|_{L^{2}}
\end{aligned}
$$

and

$$
\begin{aligned}
\left\|\nabla^{2}(m u \cdot \nabla u)\right\|_{L^{2}} & \leq C\left\|\left|\nabla^{2}(m u)\right||\nabla u|\right\|_{L^{2}}+C\left\|\left|\nabla(m u)\left\|\nabla^{2} u \mid\right\|_{L^{2}}+C\left\|\nabla^{3} u\right\|_{L^{2}}\right.\right. \\
& \leq C+C\left\|\nabla^{2}(m u)\right\|_{L^{2}}\|\nabla u\|_{H^{2}}+C\|\nabla(m u)\|_{L^{3}}\left\|\nabla^{2} u\right\|_{L^{6}}
\end{aligned}
$$




$$
\begin{aligned}
& \leq C+C\left\|\nabla^{2} m\right\|_{L^{2}}\|u\|_{L^{\infty}}+C\|\nabla m\|_{L^{6}}\|\nabla u\|_{L^{3}}+C\left\|\nabla^{2} u\right\|_{L^{2}} \\
& \leq C .
\end{aligned}
$$

Applying the $\nabla^{3}$ operator to $(1.1)_{1}$, multiplying with $\nabla^{3} m$, and integrating the resulting equation over $\mathbb{R}^{3} \times[0, T]$, we obtain

$$
\begin{aligned}
\frac{1}{2} \frac{d}{d t} \int\left|\nabla^{3} m\right|^{2} d x= & -\int_{0}^{T} \int \nabla^{3}(\operatorname{div}(m u)) \cdot \nabla^{3} m d x d t \\
\leq & C\left(\left\|\left|\nabla^{3} u\right||\nabla m|\right\|_{L^{2}}+\left\|\left|\nabla^{2} u\left\|\nabla^{2} m\left|\left\|_{L^{2}}+\right\|\right| \nabla u|| \nabla^{3} m \mid\right\|_{L^{2}}\right.\right.\right. \\
& \left.+\left\|\nabla^{4} u\right\|_{L^{2}}\right)\left\|\nabla^{3} m\right\|_{L^{2}} \\
\leq & C\left(\left\|\nabla^{3} u\right\|_{L^{2}}\|\nabla m\|_{H^{2}}+\left\|\nabla^{2} u\right\|_{L^{3}}\left\|\nabla^{2} m\right\|_{L^{6}}\right. \\
& \left.+\left\|\nabla^{3} m\right\|_{L^{2}}\|\nabla u\|_{L^{\infty}}\right)\left\|\nabla^{3} m\right\|_{L^{2}} \\
& +C\left(1+\left\|\nabla^{2} u_{t}\right\|_{L^{2}}+\left\|\nabla^{3} m\right\|_{L^{2}}\right)\left\|\nabla^{3} m\right\|_{L^{2}} \\
\leq & C+C\left\|\nabla^{2} u_{t}\right\|_{L^{2}}^{2}+C\left\|\nabla^{3} m\right\|_{L^{2}}^{2} .
\end{aligned}
$$

Similarly, we have

$$
\frac{1}{2} \frac{d}{d t} \int\left|\nabla^{3} n\right|^{2} d x \leq C+C\left\|\nabla^{2} u_{t}\right\|_{L^{2}}^{2}+C\left\|\nabla^{3} n\right\|_{L^{2}}^{2}
$$

where we have used (2.105). From (2.108)-(2.109), we have

$$
\frac{d}{d t}\left(\left\|\nabla^{3} m\right\|_{L^{2}}^{2}+\left\|\nabla^{3} n\right\|_{L^{2}}^{2}\right) \leq C+C\left\|\nabla^{2} u_{t}\right\|_{H^{2}}^{2}+C\left(\left\|\nabla^{3} m\right\|_{L^{2}}^{2}+\left\|\nabla^{3} n\right\|_{L^{2}}^{2}\right) .
$$

Then by using Gronwall's inequality and (2.104), we can get

$$
\sup _{0 \leq t \leq T}\left(\left\|\nabla^{3} m\right\|_{L^{2}}+\left\|\nabla^{3} n\right\|_{L^{2}}\right) \leq C
$$

Collecting the estimates (2.105)-(2.101), we obtain

$$
\int_{0}^{T}\|\nabla u\|_{H^{3}}^{2} d t \leq C
$$

This completes the proof of Proposition 2.7.

Proposition 2.8 ([22]) If $(m, n, u)$ is a classical solution of (1.1)-(1.3) in $[0, T]$, we have the following estimates:

$$
\sup _{0 \leq t \leq T} \sigma\left(\left\|\nabla^{2} u_{t}\right\|_{L^{2}}+\left\|\nabla^{4} u\right\|_{L^{2}}\right)+\int_{0}^{T} \sigma^{2}\left\|\nabla u_{t t}\right\|_{L^{2}}^{2} d t \leq C .
$$

\section{Proof of Theorem 1.1}

We now give the main result of this paper with the estimates in Section 2. From the local existence results, there exists a $T^{*}$ such that (1.1)-(1.4) have a unique classical solution $(m, n, u)$ on $\left(0, T^{*}\right]$. 
From Lemma 2.6 and Propositions 2.2 and 2.3, we know that there exists a $\tilde{T} \in\left(0, T^{*}\right]$ such that (2.5)-(2.6) hold for $T=\tilde{T}$. Set

$$
\bar{T}=\sup \{T \mid(2.5) \text { and (2.6) hold }\} \text {, }
$$

then $\bar{T} \geq \tilde{T}>0$. From Propositions 2.7 and 2.8 and the standard embedding

$$
L^{\infty}\left(\tau, T ; H^{1}\right) \cap H^{1}\left(\tau, T ; H^{-1}\right) \hookrightarrow C\left([\tau, T]: L^{q}\right), \quad \text { for any } q \in[2,6),
$$

we have $\nabla u_{t}, \nabla^{3} u \in C\left([\tau, T] ; L^{2} \cap L^{4}\right), \nabla u, \nabla^{2} u \in C\left([\tau, T] ; L^{2} \cap C(\bar{\Omega})\right)$. From (2.81), (2.96), and (2.113) we can obtain $\int_{\tau}^{T}\left\|\left(m\left|u_{t}\right|^{2}\right)_{t}\right\|_{L^{1}} d t \leq C$, which implies $m^{\frac{1}{2}} u_{t} \in C\left([\tau, T] ; L^{2}\right)$. This together with (3.2) gives $m^{\frac{1}{2}} \dot{u}, \nabla \dot{u} \in C\left([\tau, T] ; L^{2}\right)$.

Now, we claim that $\bar{T}=\infty$. Otherwise, $\bar{T}<\infty$. Then by Lemma 2.3 together with the estimates (2.14), (2.60) and the estimates in Propositions 2.4-2.8, and Corollaries 2.2 and 2.3, we see that $m(x, \bar{T}), n(x, \bar{T}), u(x, \bar{T})$ satisfy (1.10)-(1.12) with $g(x)=\dot{u}(x, \bar{T})$. Then, from the local existence results, there exists $\bar{T}^{\prime}>\bar{T}$ such that (2.5) and (2.6) hold for $T=\bar{T}^{\prime}$, which contradicts (3.1). Hence, we can obtain $\bar{T}=\infty$.

\section{Competing interests}

The authors declare that they have no competing interests.

\section{Authors' contributions}

All authors contributed equally to the writing of this paper. All authors read and approved the final manuscript.

\section{Author details}

'College of Science, Zhongyuan University of Technology, Zhengzhou, 450007, P.R. China. ${ }^{2}$ School of Mathematics and Information Science, Henan Polytechnic University, Jiaozuo, 454000, P.R. China. ${ }^{3}$ School of Mathematics, Northwest University, Xi'an, 710127, P.R. China.

\section{Acknowledgements}

The authors are supported by NNSFC (No. 11201371). The first and second authors are supported by NNSFC (No. 11331005) and SRDPC (20136101110015).

Received: 10 November 2014 Accepted: 10 July 2015 Published online: 08 August 2015

\section{References}

1. Brennen, CE: Fundamentals of Multiphase Flow. Cambridge University Press, New York (2005)

2. Ishii, M: Thermo-Fluid Dynamic Theory of Two-Phase Flow. Eyrolles, Paris (1975)

3. Prosperetti, A, Tryggvason, G (eds.): Computational Methods for Multiphase Flow. Cambridge University Press, New York (2007)

4. Zuber, N, Findlay, J: Average volumetric concentration in two-phase system. J. Heat Transf. 87, 453-468 (1965)

5. Bresch, D, Desjardins, B, Ghidaglia, JM, Grenier, E: Global weak solutions to a generic tow-fluid model. Arch. Ration. Mech. Anal. 196, 599-629 (2010)

6. Evje, S, Karlsen, $\mathrm{KH}$ : Global weak solutions for a viscous liquid-gas model with singular pressure law. Commun. Pure Appl. Anal. 8, 1867-1894 (2009)

7. Evje, S, Flåtten, T, Friis, HA: Global weak solutions for a viscous liquid-gas model with transition to single-phase gas flow and vacuum. Nonlinear Anal. 70, 3864-3886 (2009)

8. Yao, L, Zhu, CJ: Free boundary value problem for a viscous two-phase model with mass-dependent viscosity. J. Differ. Equ. 247, 2705-2739 (2009)

9. Yao, L, Zhu, CJ: Existence and uniqueness of global weak solution to a two-phase flow model with vacuum. Math. Ann. 349, 903-928 (2011)

10. Evje, S, Karlsen, KH: Global existence of weak solutions for a viscous two-phase model. J. Differ. Equ. 245, 2660-2703 (2008)

11. Evje, S: Global weak solutions for a compressible gas-liquid model with well-formation interaction. J. Differ. Equ. 251, 2352-2386 (2011)

12. Evje, S: Weak solutions for a gas-liquid model relevant for describing gas-kick in oil wells. SIAM J. Math. Anal. 43 , 1887-1922 (2012)

13. Friis, HA, Evje, S: Global weak solutions for a gas-liquid model with external forces and general pressure law. SIAM J. Appl. Math. 71, 409-442 (2011)

14. Yao, L, Zhang, T, Zhu, CJ: Existence and asymptotic behavior of global weak solutions to a 2D viscous liquid-gas two-phase flow model. SIAM J. Math. Anal. 42, 1874-1897 (2010) 
15. Wen, HY, Yao, L, Zhu, CJ: A blow-up criterion of strong solution to a 3D viscous liquid-gas two-phase flow model with vacuum. J. Math. Pures Appl. 97, 204-229 (2012)

16. Yao, L, Zhang, T, Zhu, CJ: A blow-up criterion for a 2D viscous liquid-gas two-phase flow model. J. Differ. Equ. 250, 3362-3378 (2011)

17. Yao, L, Yang, J, Guo, ZH: Blow-up criterion for 3D viscous liquid-gas two-phase flow model. J. Math. Anal. Appl. 395, 175-190 (2012)

18. Hao, CC, Li, HL: Well-posedness for a multi-dimensional viscous liquid-gas tow-phase flow model. SIAM J. Math. Anal. 44, 1304-1332 (2012)

19. Bresch, D, Huang, XD, Li, J: Global weak solutions to one-dimensional non-conservative viscous compressible two-phase system. Commun. Math. Phys. 309, 737-755 (2012)

20. Guo, ZH, Yang, J, Yao, L: Global strong solution for a 3D viscous liquid-gas two-phase flow model with vacuum. J. Math. Phys. 52, 093102 (2011)

21. Yao, L, Yang, J, Guo, ZH: Global classical solution for a three-dimensional viscous liquid-gas two-fluid flow model with vacuum. Acta Math. Appl. Sin. (Engl. Ser.) 30, 989-1006 (2014)

22. Duan, Q: Global well-posedness of classical solutions to the compressible Navier-Stokes equations in a half-space. J. Differ. Equ. 253, 167-202 (2012)

23. Cho, Y, Kim, H: On classical solution of the compressible Navier-Stokes equations with nonnegative initial densities. Manuscr. Math. 120, 91-129 (2006)

24. Huang, XD, Li, J, Xin, ZP: Global well-posedness of classical solutions with large oscillations and vacuum to the three-dimensional isentropic compressible Navier-Stokes equations. Commun. Pure Appl. Math. 65, 549-585 (2012)

25. Hoff, D: Compressible flow in a half-space with Navier boundary conditions. J. Math. Fluid Mech. 7, 315-338 (2005)

26. Perepelitsa, M: Weak solutions of the Navier-Stokes equations for compressible flows in a half-space with no-slip boundary conditions. Arch. Ration. Mech. Anal. 212, 709-726 (2014)

27. Cho, Y, Kim, H: Existence results for viscous polytropic fluid with vacuum. J. Differ. Equ. 228, 377-411 (2006)

28. Zlotnik, AA: Uniform estimates and stabilization of symmetric solutions of a system of quasilinear equations. Differ. Equ. 36, $701-716(2000)$

29. Simon, J: Nonhomogeneous viscous incompressible fluids: existence of velocity, density and pressure. SIAM J. Math. Anal. 21, 1093-1117 (1990)

30. Hoff, D: Global solutions of the Navier-Stokes equations for multidimensional compressible flow with discontinuous initial data. J. Differ. Equ. 120, 215-254 (1995)

31. Zhang, T: Global solution of compressible Navier-Stokes equation with a density-dependent viscosity coefficient. J. Math. Phys. 52, 043510 (2011)

\section{Submit your manuscript to a SpringerOpen ${ }^{\circ}$ journal and benefit from:}

- Convenient online submission

Rigorous peer review

- Immediate publication on acceptance

- Open access: articles freely available online

- High visibility within the field

- Retaining the copyright to your article 